\title{
P-Chirogenic silylphosphine-boranes: synthesis and phospha-Michael reactions
}

\author{
Eric Bonnefille, Arnaud Tessier, ${ }^{\dagger *}$ Hélène Cattey, Pierre Le Gendre and Sylvain Jugé* \\ Institut de Chimie Moléculaire de l'Université de Bourgogne (ICMUB-StéréochIM-UMR CNRS \\ 6302), 9 avenue A. Savary BP47870, 21078 Dijon Cedex, France \\ ${ }^{\dagger}$ Present adress: Université de Nantes, CNRS, CEISAM, UMR CNRS 6230, Faculté des Sciences \\ et des Techniques, 2 rue de la Houssinière, BP 92208, 44322 Nantes Cedex 3, France \\ E-mail: Arnaud.Tessier@univ-nantes.fr, sylvain.juge@u-bourgogne.fr
}

Dedicated to Prof. Jürgen Martens for his $65^{\text {th }}$ birthday

DOI: $\underline{\text { http://dx.doi.org/10.3998/ark.5550190.p009.189 }}$

\begin{abstract}
Chiral and achiral silylphosphine-boranes were prepared in high yields by reaction of phosphide boranes with halogenosilanes. Their reaction at room temperature with Michael acceptors afforded 1,4-addition products as silylenol ether or ketone derivatives in good to excellent yields. In the case of the 2,3-dihalogeno-maleimides, the double addition of silylphosphine-borane led to the corresponding trans-diphosphine-boranes in $86 \%$ yield. Noteworthy, the reaction of Pchirogenic silylphosphine-boranes with enones afforded the phospha-Michael adducts without racemization at the P-center. While the silylphosphine-boranes have been scarcely described so far, these compounds demonstrate their great interest for the synthesis of chiral and achiral functionalized organophosphorus compounds.
\end{abstract}

Keywords: Phosphine-borane, silylphosphine, phospha-Michael reaction, P-chirogenic phosphine, silylenol ether.

\section{Introduction}

Organophosphorus chemistry ${ }^{1}$ is a very active research field that concerns numerous applications in agrochemistry, ${ }^{2}$ health, ${ }^{3,4}$ biology, ${ }^{5}$ materials ${ }^{6}$ and additives, ${ }^{7}$ hydrometallurgy, ${ }^{8}$... Chiral organophosphorus compounds are also of particular interest because their properties often depend on their configuration. ${ }^{2-5}$ Indeed, they played a significant role as ligands in metal based asymmetric catalysis ${ }^{9}$ as well as Brönsted acid or Lewis bases in organocatalysis. ${ }^{10}$ Usually, the stereoselective synthesis of chiral organophosphorus compounds with P-C bond formation was 
performed using chlorophosphines or phosphides as electrophilic or nucleophilic reagents, respectively. ${ }^{9,11}$ In the last decade, the asymmetric hydrophosphination ${ }^{12,13}$ and phospha-Michael addition ${ }^{14}$ have also emerged as powerful methodologies for the synthesis of functional derivatives such as chiral organophosphorus compounds, that hold promise for applications in asymmetric catalysis. In this last case, typical reactions of Michael acceptor with free secondary phosphines or their oxide, sulfur or other borane derivatives, were achieved either in basic conditions or by heating. ${ }^{14,15}$ On the other hand, the asymmetric phospha-Michael addition could also be performed using chiral transition metal catalysts ${ }^{16-18}$ or organocatalysts. ${ }^{19-21}$ Among the nucleophilic phosphorus reagents, the silylphosphines have recently retained the attention because these compounds are considered more electron-rich than the parent secondary phosphines due to the electrodonating effect of the silicon moiety. ${ }^{22,23}$ Usually, the silylphosphines react with electrophiles through nucleophile-induced activation, ${ }^{24,25}$ activated electrophile-driven reactions, ${ }^{26-29}$ or using transition metal catalysis. ${ }^{30-33}$ Thus, the silylphosphines $\mathbf{1}$ and $\mathbf{2}$ have been used for the stereoselective synthesis of MalPHOS $\mathbf{5}$ and Pchirogenic phosphines $\mathbf{6}$, by double phospha-Michael addition with the 2,3-dichloromaleic anhydride $3,{ }^{34}$ or Pd-catalyzed enantioselective arylation of the iodo compound $4,{ }^{35}$ respectively (Scheme 1a,b).<smiles>C[C@@H]1CC[C@@H](C)P1[AsH3]</smiles>

1

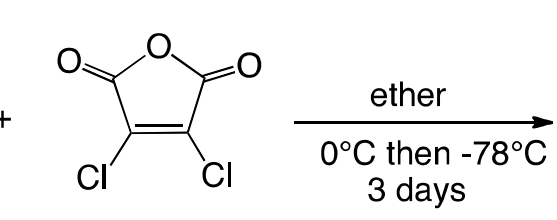

3

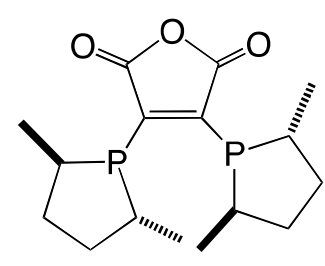

5 53\% yield (a)<smiles>[B][PH](C)([18OH])c1ccccc1C(=O)Nc1ccccc1</smiles>

6 yield $53 \%$; e.e $98 \%$

\section{Scheme 1}

While in the last decades the use of borane as $\mathrm{P}(\mathrm{III})$-protecting group has resulted in significant breakthroughs for the stereoselective synthesis of tricoordinated organophosphorus compounds, surprisingly the silylphosphine-borane complexes have been scarcely studied. ${ }^{36}$ As part of our on-going program on the stereoselective synthesis of P-chirogenic organophosphorus compounds, we recently reported a new method for the preparation of Pchirogenic phosphide-boranes that involves metal-halide exchange of the corresponding chlorophosphines. $^{37,38}$ This method, which proceeds with retention of configuration at the Pcenter, was used for the synthesis of P-chirogenic phosphines by reaction with alkyl halide or 
aryne reagents. ${ }^{37,38}$ These results led us to envisage the synthesis and study of silylphosphineboranes. Herein we report the first examples of P-chirogenic silylphosphane-boranes and their application to the stereoselective synthesis of functionalized phosphine-boranes by phosphaMichael addition under mild uncatalyzed conditions.

\section{Results and Discussion}

The silylphosphine-boranes 11a,b were prepared in $80-93 \%$ yields by reaction of phosphideboranes 9 , previously obtained by deprotonation of the secondary diphenylphosphine- borane 7a, with the corresponding halogenosilane 10a or 10b (Scheme 2). After removal of the solvent, the residue was dissolved in toluene, then filtered to afford the silylphosphine-boranes 11a,b which were used without further purification. In these conditions, when the P-chirogenic $(S)$-o-anisylor $(R)$-ferrocenylphosphine-boranes $\mathbf{7 c}, \mathbf{d}$, previously prepared from the chlorophosphine-boranes $\mathbf{8 c}, \mathbf{d},{ }^{37,38}$ were used, the corresponding silylphosphine-boranes 11c,d were obtained with ee up to $87 \%$, by reaction with TMSBr 10c (Scheme 2). As the deprotonation of secondary P-chirogenic phosphine-boranes $\mathbf{7 c , d}$ and their reactions proceed with retention of configuration at the phosphorus center, ${ }^{37,38}$ it is reasonable to think that silylation with TMSBr 10c follows the same stereochemistry. All silylphosphine-boranes $\mathbf{1 1}$ could be purified by chromatography, but in low isolated yields. Therefore, they were better used immediately after preparation without further purification.

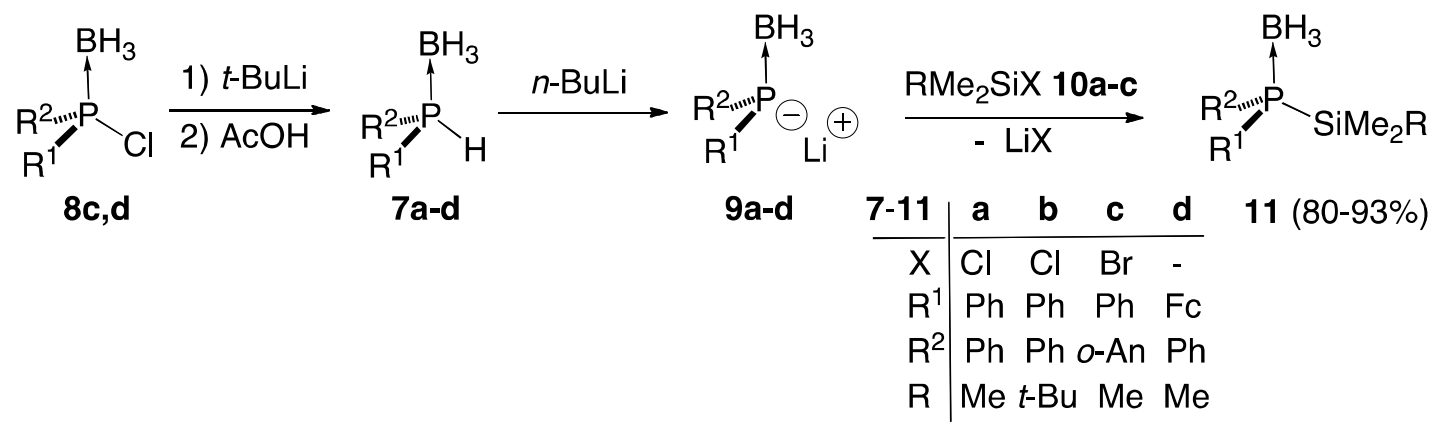

\section{Scheme 2}

Firstly, the reactivity of the silylphosphine-borane 11a was investigated in the Michael addition to enones 13 by comparison with the free trimethylsilylphosphine $\mathbf{1 2}$ (Scheme 3). When the trimethylsilylphosphine 12 was stirred with the enone 13a (or 13b) in THF during 16 hours at room temperature, the $\beta$-phosphinoketone 14a (or 14b) was obtained after purification by chromatography on silica gel in $51 \%$ (or $58 \%$ ) yield (Scheme 3 ). ${ }^{39}$ 


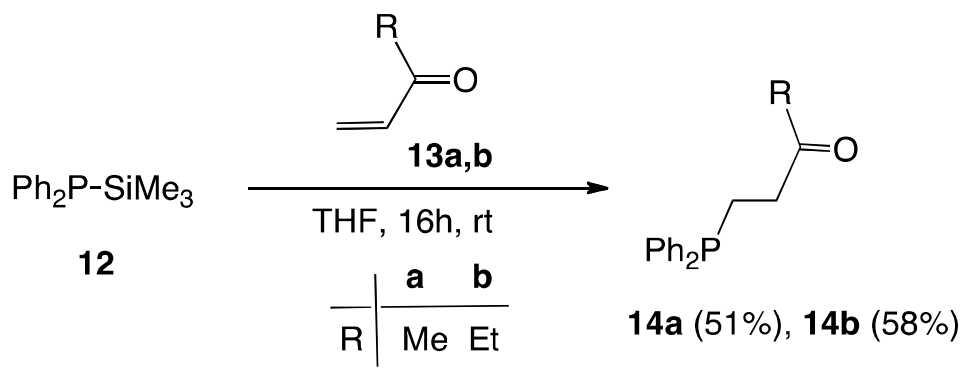

\section{Scheme 3}

Surprisingly, when the silylphosphine-borane 11a was used in the same conditions the reaction with enones 13a,b led to the corresponding trimethylsilylenol ethers $\mathbf{1 5 a}$ (or 15b) as an isomeric mixture in 2:1 ratio and with yields up to $48 \%$ (Table 1, entries 1,2 ). In the case where the silylphosphine-borane 11a was reacted with cyclohexenone 13c in THF or toluene, the silylenol ether 15c was successfully isolated in 84 or $63 \%$ yields (entries 3,4). Similarly, the reaction of the $t$-butyldimethylsilyl phosphine-borane $\mathbf{1 1 b}$ with the cyclohexenone $\mathbf{1 3 c}$ led to the corresponding silylenol ether $\mathbf{1 5 d}$ in $77 \%$ yield (entry 5).

Table 1. Reaction of silylphosphine-boranes $\mathbf{1 1}$ with Michael acceptors $\mathbf{1 3}$

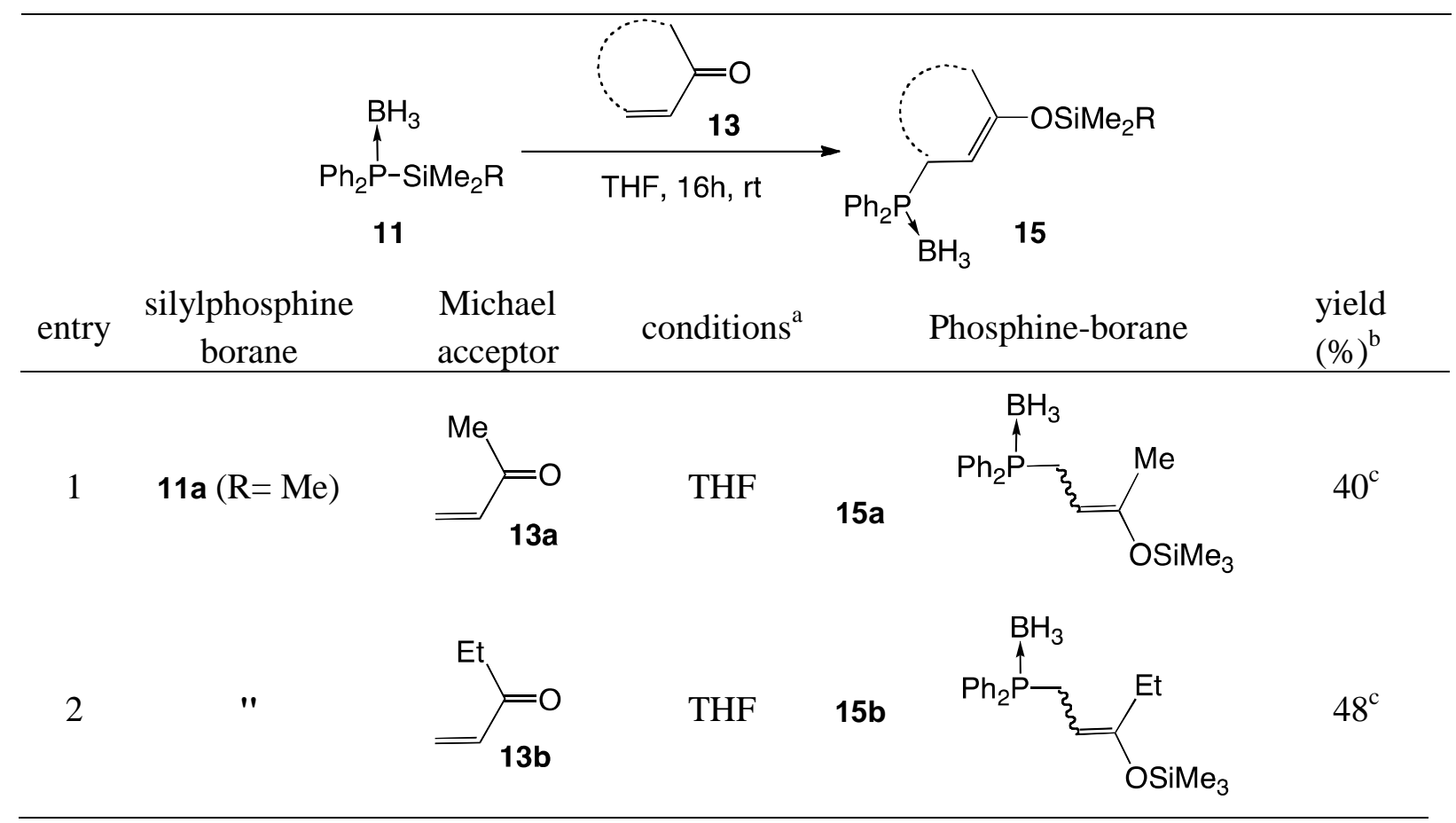


Table 1 (continued)

\begin{tabular}{|c|c|c|c|c|c|c|}
\hline entry & $\begin{array}{l}\text { silylphosphine } \\
\text { borane }\end{array}$ & $\begin{array}{l}\text { Michael } \\
\text { acceptor }\end{array}$ & conditions $^{\mathrm{a}}$ & & Phosphine-borane & $\begin{array}{l}\text { yield } \\
(\%)^{\mathrm{b}}\end{array}$ \\
\hline 3 & $"$ & $\begin{array}{l}=0 \\
13 c\end{array}$ & THF & $15 c$ & OSi & 84 \\
\hline 4 & $"$ & $13 c$ & Toluene & $15 c$ & $\mathrm{H}_{3}$ & 63 \\
\hline 5 & $11 \mathrm{~b}(\mathrm{R}=t-\mathrm{Bu})$ & $13 c$ & THF & $15 d$ & osin & 77 \\
\hline
\end{tabular}

${ }^{a}$ Reaction at $\mathrm{rt}$ for 16 hours. ${ }^{\mathrm{b}}$ Isolated yield. ${ }^{\mathrm{c}}$ Obtained as an isomeric mixture in 2:1 ratio.

Interestingly, treatment of phosphine-borane 15c with DABCO led quantitatively to the corresponding free phosphine silylenol ether $\mathbf{1 6}$ by decomplexation of the borane moiety (Scheme 4).

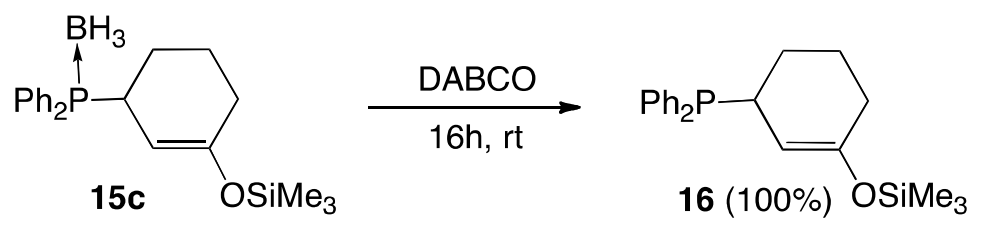

\section{Scheme 4}

On the other hand, when the reaction of cyclohexenone 13c was performed with $(S)$ ferrocenylphenyl(trimethylsilyl)phosphine-borane 11d ( $85 \%$ ee), the silylenol ether 15e was obtained as an epimeric mixture in $1: 1$ ratio in $75 \%$ yield (Table 2, entry 1 ). In the case where the $(R)$-o-anisylphenyl(trimethylsilyl)phosphine-borane 11c was reacted with the enone 13a, the $\beta$-(boranato)phosphinoketone 14c was obtained in $72 \%$ yield and with $82 \%$ ee (entry 2). Similarly, the reaction of $(S)$-ferrocenylphenyl(trimethylsilyl)phosphine-borane 11d, prepared from secondary phosphine-borane $\mathbf{7 d}(33 \%$ ee), with the enone $\mathbf{1 3 b}$, led to the $\beta$ (boranato)phosphinoketone 14d in $95 \%$ yield and with $33 \%$ ee (entry 3 ). In these cases, the formation of the ketone derivatives $\mathbf{1 4 c}, \mathbf{d}$ was explained by an easier hydrolysis of the silylenol intermediates in the conditions of the reaction (entries 2,3). 
Table 2. Michael-addition of P-chirogenic silylphosphines 11c,d with enone 13a-c

\begin{tabular}{|c|c|c|c|c|c|c|}
\hline entry & $\begin{array}{l}\text { sec-phosphine } \\
\text { boranes (ee \%) }\end{array}$ & $\begin{array}{l}\text { silylphosphine } \\
\text { boranes }\end{array}$ & enones & conditions & products $(\text { ee } \%)^{\mathrm{a}}$ & $\begin{array}{l}\text { yield } \\
(\%)^{\mathrm{b}}\end{array}$ \\
\hline 1 & 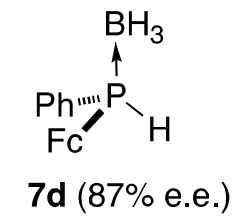 & $\mathrm{Fc}_{\mathbf{1 1 d}}^{\mathrm{Ph}^{\mathrm{B} \mathrm{H}_{3}}} \mathrm{SiMe}_{3}$ & $13 c$ & Toluene & 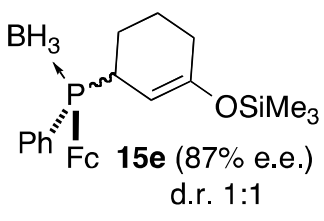 & 75 \\
\hline 2 & 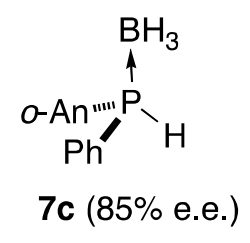 & 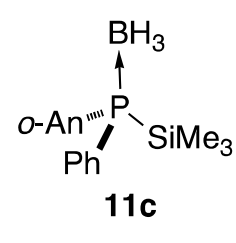 & $13 a$ & THF & o-An"il & 72 \\
\hline 3 & 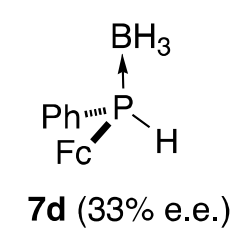 & 11d & $13 b$ & Toluene & 14d (33\% e.e.) & 95 \\
\hline
\end{tabular}

${ }^{\mathrm{a}}$ Determined by HPLC on chiral column. ${ }^{\mathrm{b}}$ Isolated yield.

Interestingly, the phospha-Michael additions of Table 2 proceeded without racemization as the enantiomeric excesses of $\mathbf{1 5 e}, \mathbf{1 4 c}$, and $\mathbf{1 4 d}$, were close to those of the corresponding secondary phosphines $\mathbf{7 c}$ and $\mathbf{7 d}$ used for the preparation of the intermediate silyl phosphines 11c and 11d, respectively (entry 1). While the absolute configuration of the products $\mathbf{1 4 c}, \mathbf{d}$ or $\mathbf{1 5 e}$ was not established, we believe that the reaction proceeds with a concerted mechanism involving retention of configuration at the P-center as showed in Scheme 5b.

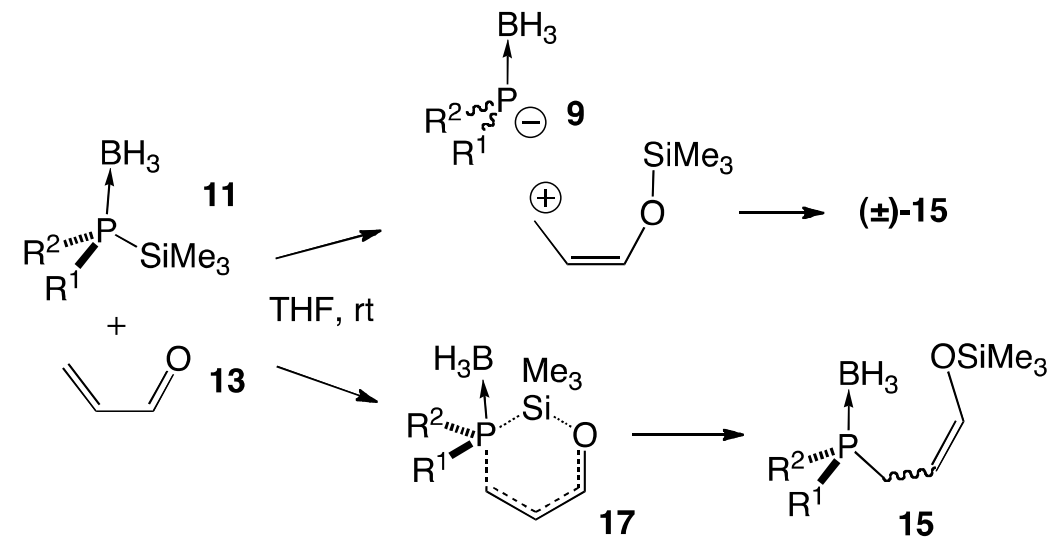

(a)

\section{Scheme 5}


Indeed, in the case where the mechanism would lead to the formation of P-chirogenic phosphide-borane 9 by nucleophilic attack of the enone 13 first on the silyl group, a racemized product $\mathbf{1 5}$ would be obtained due to the poor configurational stability of $\mathbf{9}$ at room temperature (Scheme 5a). ${ }^{37,38}$ On the contrary, when a concerted transition state $\mathbf{1 7}$ is formed by interaction of the enone 13 with the silyphosphine-borane 11 both on the Si- and P-atoms, precisely in anti position of the P-B bond, the product $\mathbf{1 5}$ is obtained with retention of configuration at the phosphorus center (Scheme 5b).

Finally, the 1,4-addition of silylphosphine-boranes $\mathbf{1 1}$ was investigated with various kinds of electrophilic acceptors such as methyl propiolate 13d, 2,3-dihalogeno-maleimide 13e,f and quinoxaline derivatives 13g. In the case of the methyl propiolate 13d, the reaction with the silylphosphine-borane 11a led to the (boranato)phosphine-enoate 18, which is stereoselectively obtained as $(E)$-isomer in $56 \%$ isolated yield (Table 3, entry 1 ).

Table 3. Addition of silylphosphine-borane 11a to electrophilic acceptors 13d-g

$\begin{array}{llllll}\text { entry } & \begin{array}{c}\text { silylphosphine } \\ \text { (borane) }\end{array} & \text { Michael acceptor } & \text { Conditions }^{\mathrm{a}} & \mathrm{Yield}^{\mathrm{b}} \\ (\%)\end{array}$


Table 3 (continued)

\begin{tabular}{|c|c|c|c|c|c|}
\hline entry & $\begin{array}{l}\text { silylphosphine } \\
\text { (borane) }\end{array}$ & Michael acceptor & Conditions $^{\mathrm{a}}$ & product & $\begin{array}{c}\text { Yield }^{\mathrm{b}} \\
(\%)\end{array}$ \\
\hline \multirow[t]{2}{*}{8} & $"$ & & THF & & 41 \\
\hline & & $13 g$ & & 21 & \\
\hline 9 & $"$ & $13 g$ & Toluene & 21 & 46 \\
\hline
\end{tabular}

${ }^{\mathrm{a}}$ Reaction at rt for 16 hours. ${ }^{\mathrm{b}}$ Isolated yield.

When the reaction of silylphosphine-borane 11a was performed with 2,3-dichloromaleimide 13e in THF, the 2,3-di(boranato)phosphinosuccinimide ( \pm )-19a was obtained in $47 \%$ yield (entry 2). Crystals of diphosphine-diborane 19a have been obtained and an OLEX view of the X-ray structure is depicted in Figure 1. The compound crystallizes in the $\mathrm{C} 2 / \mathrm{c}$ space group with both enantiomers in the unit cell (i.e. racemate). The structure of 19a is chiral and exhibits a $\mathrm{C}_{2}$ crystallographic axis spanning the ring and both (boranato)diphenylphosphino groups, which are in trans relative configuration (Figure 1).

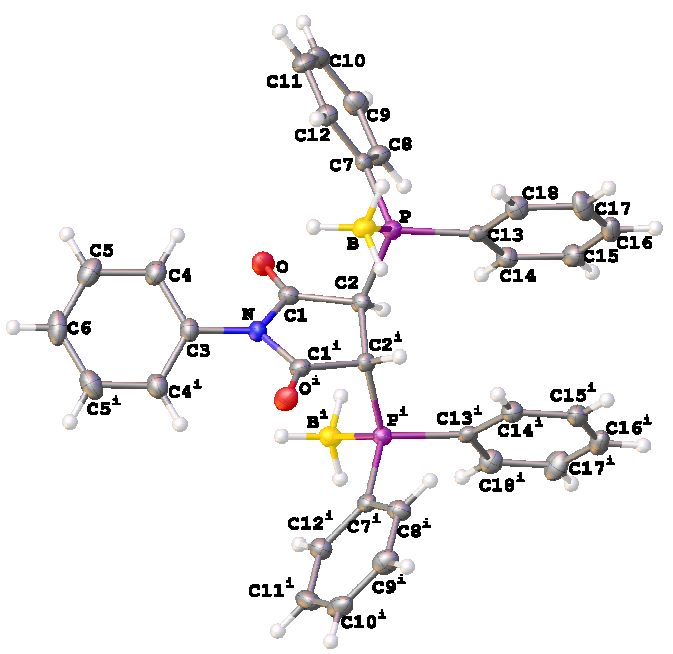

Figure 1. $\mathrm{OLEX}^{40}$ view of the compound 19a. Symmetry transformations used to generate equivalent atoms (i): 1-x, y, 3/2-z. Selected bond lengths $[\AA]$, angles $\left[{ }^{\circ}\right]$ and dihedral angles $\left[{ }^{\circ}\right]$ : P-B 1.910(2); C2-P 1.8577(18), C2-C2' 1.545(3); C7-P-C2 105.82(8), C13-P-C2 104.54(8), C13P-C7 106.48(8); C2-P-B 110.54(9); C1-C2-P-B 71.23(14), N-C1-C2-P -100.70(12), C1-C2-P$\mathrm{C} 13-163.77(12)$. C2-C1-N-C1 ${ }^{\mathrm{i}}-7.28(8)$.

On the other hand, when the silylphosphine-borane 11a was added to the dichloromaleimide 13e in $\mathrm{Et}_{2} \mathrm{O}$, the (monoboranato)diphosphinomaleimide derivative 20a was isolated as major product (35\% yield, entry 3). By contrast, when the reaction was performed in toluene the 2,3- 
di(boranato)phosphinosuccinimide 19a and the maleimide derivative 20a were obtained in 88 and $11 \%$ yields, respectively (entry 4 ). Similarly, the addition of silylphosphine-borane 11a to the dibromomaleimide $13 \mathrm{f}$ in THF led to the 2,3-di(boranato)phosphinosuccinimide 19b in 50\% yield (entry 5). When the reaction was run in $\mathrm{Et}_{2} \mathrm{O}$, the (monoboranato)diphosphinomaleimide 20b was obtained as major product (37\% yield, entry 6). Finally, when the reaction of 11a with the 2,3-dibromomaleimide $\mathbf{1 3 f}$ was performed in toluene, the maleimide derivative $\mathbf{2 0 b}$ was obtained in $96 \%$ yield (entry 7).

The formation of the succinimide or maleimide derivatives 19 (or 20) could be explained by two possible pathways via the intermediate $\mathbf{2 3}$ depending on the substrate $\mathbf{1 3}$, the solvent and the halide. The compound $\mathbf{2 3}$ was formed by addition of two equivalents of silylphosphine-borane 11a to the dihalogenomaleimide 13e (or 13f), via the diphenylphosphine-borane 22 (Scheme 6). The intermediate $\mathbf{2 3}$ can evolve either towards the formation of the bis-silylether derivative $\mathbf{2 4}$ by reaction with a trimethylsilyl reagent (e.g. TMSX), or the conventional double Michael-addition product 25 (Scheme 6). Finally, the hydrolysis of $\mathbf{2 4}$ and the loss of a borane moiety due to steric congestion in compound 25 led to the succinimide and maleimide products 19 and 20 , respectively (Scheme 6).

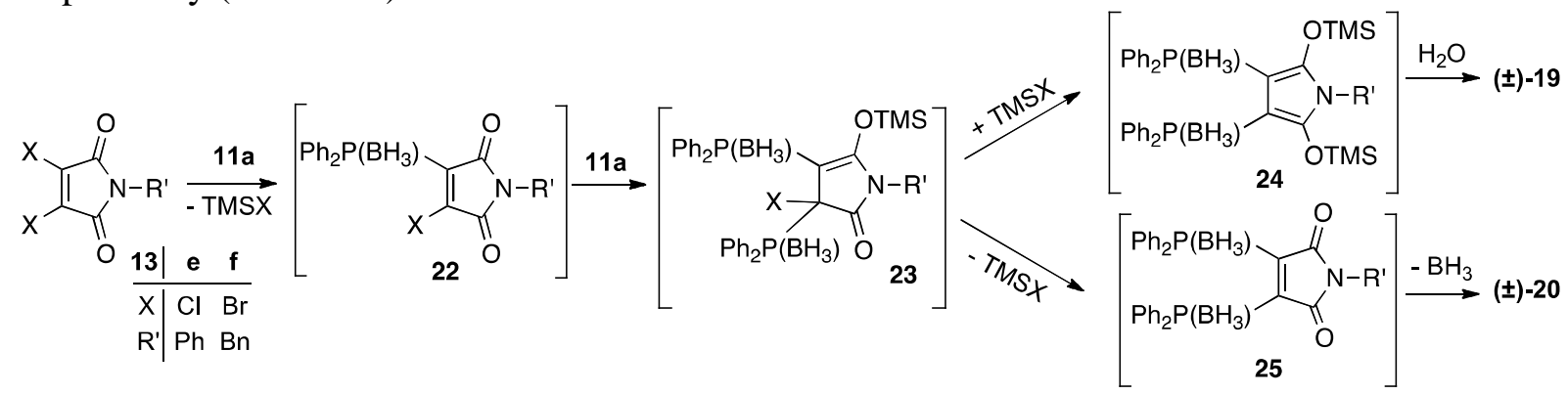

\section{Scheme 6}

On the other hand, when the dichloroquinoxaline 13g was used as electrophilic acceptor, the reaction with the silylphosphine-borane 11a in THF (or toluene) led to the monophosphine product 21 in 41 or $46 \%$ yield, respectively (entries 8,9). The formation of compound 21 was explained by only one addition of silylphosphine-borane 11a to the 2,3-dichloroquinoxaline substrate 18 and the decomplexation of borane due to steric hindrance of the 2-chloroquinoxaline substituent. The structure of compound 21 was established by X-ray analysis (Figure 2). This structure shows the chloroquinoxaline substituent in staggered conformation with respect to both phenyl groups borne by the phosphorus atom, the chlorine atom facing the lone pair of the phosphorus atom (Figure 2) 


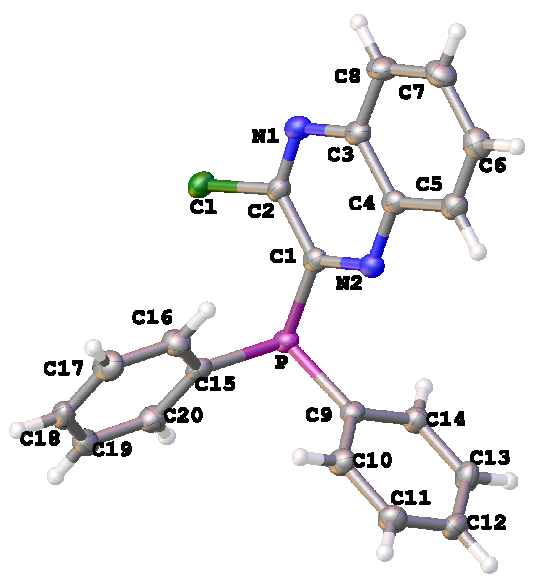

Figure 2. $\mathrm{OLEX}^{40}$ view of the compound 21. Selected bond lengths $[\AA$ A $]$, angles $\left[{ }^{\circ}\right]$ and dihedral angles [ ${ }^{\circ}$ ]: C1-P 1.844(3); C9-P 1.822(2), C15-P 1.828(3); C1-C2 1.433(3); C2-Cl 1.741(3); N2C1-P 120.70(18), C9-P-C1 102.30(11), C9-P-C15 103.87(11); Cl-C2-C1-P 7.0(3), C2-C1-PC9 175.4(2), C2-C1-P-C15 -77.3(2).

\section{Conclusions}

The silylphosphine-boranes were prepared in high yields by reaction of phosphide boranes, previously obtained either by deprotonation of the secondary phosphine-boranes or by metal halide exchange of the chlorophosphine-boranes with halogenosilanes. The reaction of silylphosphine-boranes with various Michael-acceptors led to the addition products in yields up to $96 \%$ under uncatalyzed mild conditions. In the case of the reaction with enones the product are mainly isolated as silylenol ether derivatives. Moreover, the silylphosphine-boranes also react with 2,3-dihalogenomaleimides to afford the corresponding trans diphosphine-diborane complexes in yields up to $86 \%$. The trans configuration of both phosphine-borane moieties has been established by X-ray crystal structure analysis. Interestingly, when P-chirogenic silylphosphine-borane were used, the reaction with enones led to the phospha-Michael products without racemization at the P-center. While the silylphosphine-boranes have been scarcely described so far, these compounds reveal a great potential for the synthesis of chiral and achiral functionalized organophosphorus compounds.

\section{Supporting information available}

NMR spectra and crystallographic data in CIF format for compounds 19a and 21. This material is available free of charge via the internet at http://www.arkat-usa.org. 


\section{Experimental Section}

All reactions were carried out under inert atmosphere. Solvents were dried and purified by conventional methods prior to use. Tetrahydrofuran (THF) and toluene were distilled from sodium/benzophenone and stored under argon. Diphenyl(trimethylsilyl)phosphine $\mathbf{1 2}$ was purchased from commercial sources and used without purification. The P-chirogenic secondary phosphine-boranes $(S)$-7c and $(R)$-7d were prepared using the (-)- and (+)-ephedrine methodology, respectively. ${ }^{37,38}$ Reactions were monitored by thin-layer chromatography (TLC) using $0.25 \mathrm{~mm}$ E. Merck precoated silica gel plates. Flash chromatography was performed with the indicated solvents using silica gel $60(60 \mathrm{AAC}, 35-70 \mu \mathrm{m})$. NMR spectra $\left({ }^{1} \mathrm{H},{ }^{13} \mathrm{C},{ }^{31} \mathrm{P},{ }^{29} \mathrm{Si}\right)$ were recorded on Bruker Avance 600, 500 or $300 \mathrm{MHz}$ spectrometers at ambient temperature and chemical shifts are reported in ppm using TMS as internal reference for ${ }^{1} \mathrm{H},{ }^{13} \mathrm{C}$ and ${ }^{29} \mathrm{Si}$ NMR or $85 \%$ phosphoric acid as external reference for ${ }^{31} \mathrm{P}$ NMR. The signals are reported as $\mathrm{s}=$ singlet, $\mathrm{d}=$ doublet, $\mathrm{t}=$ triplet, $\mathrm{q}=$ quartet, $\mathrm{m}=$ multiplet, br.s = broad signal, coupling constant(s) in Hertz and with their integration. The infrared spectra were recorded on a FT-IR Bruker Vector 22 and the bands are reported in $\mathrm{cm}^{-1}$. Melting points were mesured on a Kofler melting points apparatus and are uncorrected. Optical rotation values were determined at $20^{\circ} \mathrm{C}$ on polarimeter Perkin Elmer 341 at $589 \mathrm{~nm}$ (sodium lamp). HPLC analyses were performed on a chromatograph equipped with a UV detector at $\lambda=210 \mathrm{~nm}$ and $\lambda=254 \mathrm{~nm}$. High Resolution Mass Spectra (HRMS) were performed on Thermo Orbitrap XL under ESI conditions with a micro Q-TOF detector. Elemental analyses were measured on Thermo EA 1112 with a precision superior to $0.3 \%$ on a CHNS-O instrument apparatus.

\section{Crystal Structure Determination}

Diffraction data were collected on a Nonius Kappa CCD diffractometer equipped with an Oxford Cryosystems low-temperature apparatus operating at $T=115 \mathrm{~K}$. Data were measured using $\varphi$ and $\omega$ scans using $\mathrm{MoK}_{\alpha}$ radiation $(\lambda=0.71073 \AA$, X-ray tube, $50 \mathrm{kV}, 32 \mathrm{~mA})$. The total number of runs and images was based on the strategy calculation the program Collect. ${ }^{41}$ Cell parameters were retrieved using the SCALEPACK software and refined using DENZO. ${ }^{42}$ Data reduction was performed using the $\mathrm{DENZO}^{42}$ software which corrects for Lorentz polarisation. The structure was solved by Direct Methods using the SIR92 $2^{43}$ program structure solution program and refined by Least Squares using version of the ShelXL ${ }^{44,45}$ (Sheldrick, 2008). All nonhydrogen atoms were refined anisotropically. Hydrogen atom positions were calculated geometrically and refined using the riding model. CCDC Deposition Number: Compound 19a (CCDC 1048105); Compound 21 (CCDC 1048106).

Trimethylsilyl(diphenyl)phosphine-borane (11a). ${ }^{36}$ To a solution of diphenyl phosphineborane $7 \mathbf{a}\left(\mathrm{R}^{1}=\mathrm{Ph}\right)(3.31 \mathrm{~g}, 16.6 \mathrm{mmol})$ in $30 \mathrm{~mL}$ of THF under inert atmosphere, was added dropwise at $-78{ }^{\circ} \mathrm{C} n$-BuLi $\left(11.4 \mathrm{~mL}, 18.2 \mathrm{mmol}, 1.1\right.$ equiv). After stirring 30 minutes at $-78{ }^{\circ} \mathrm{C}$, a solution of chloro(trimethyl)silane $\mathbf{1 0 a}(2.10 \mathrm{~mL}, 16.6 \mathrm{mmol})$ in $10 \mathrm{~mL}$ of THF was added. The 
reaction mixture was kept at $-78{ }^{\circ} \mathrm{C}$ during 30 minutes and was stirring at room temperature overnight. After removing the solvent under vacuum, the residue was dissolved in toluene and was filtered off. After new removal of the solvent under vacuum, 11a was obtained as a colorless uncrystallized compound $(4.21 \mathrm{~g}, 93 \%$ yield $) .{ }^{1} \mathrm{H}$ NMR $\left(300 \mathrm{MHz}, \mathrm{C}_{6} \mathrm{D}_{6}\right): \delta 0.16\left(\mathrm{~d},{ }^{3} J_{\mathrm{PH}}=6.0\right.$ $\mathrm{Hz}, 9 \mathrm{H}, \mathrm{SiMe}_{3}$ ); 0.90-2.50 (br, 3H, $\left.\mathrm{BH}_{3}\right) ; 7.01-7.08$ (m, 6H, Ph); 7.66-7.70 (m, 4H, Ph). ${ }^{13} \mathrm{C}$ $\operatorname{NMR}\left(75.4 \mathrm{MHz}, \mathrm{C}_{6} \mathrm{D}_{6}\right): \delta-2.5\left(\mathrm{~d},{ }^{2} J_{\mathrm{PC}}=9.3 \mathrm{~Hz}, \mathrm{SiMe}_{3}\right) ; 129.0\left(\mathrm{~d}, J_{\mathrm{PC}}=9.3 \mathrm{~Hz}, \mathrm{C}\right.$-aryl $) ; 130.4$ $\left(\mathrm{d}, J_{\mathrm{PC}}=2.3 \mathrm{~Hz}, \mathrm{C}\right.$-aryl $) ; 133.2\left(\mathrm{~d}, J_{\mathrm{PC}}=9.3 \mathrm{~Hz}, \mathrm{C}\right.$-aryl $) ; 133.7\left(\mathrm{~d}, J_{\mathrm{PC}}=8.2 \mathrm{~Hz}, \mathrm{C}\right.$-aryl $) .{ }^{31} \mathrm{P}$ NMR (121.4 MHz, $\left.\mathrm{C}_{6} \mathrm{D}_{6}\right): \delta-23.9\left(\mathrm{~d},{ }^{1} J_{\mathrm{PB}}=38 \mathrm{~Hz}\right) .{ }^{29} \mathrm{Si} \mathrm{NMR}\left(99.4 \mathrm{MHz}, \mathrm{C}_{6} \mathrm{D}_{6}\right): \delta+4.6\left(\mathrm{~d},{ }^{1} J_{\mathrm{SiP}}\right.$ = 44.7 Hz). IRFT (neat): 3057, 2959, 2926, 2856, 2383, 1437, 1256, 1112, 1066, 992, 846, 733, 691.

Diphenyl[t-butyl(dimethyl)silyl]phosphine-borane (11b). To a solution of diphenyl phosphine-borane $7 \mathbf{a}\left(\mathrm{R}^{1}=\mathrm{Ph}\right)(0.52 \mathrm{~g}, 2.6 \mathrm{mmol})$ in $6 \mathrm{~mL}$ of THF under inert atmosphere, was added dropwise at $-78{ }^{\circ} \mathrm{C} n$-BuLi $(1.8 \mathrm{~mL}, 2.86 \mathrm{mmol}, 1.1$ equiv). After stirring 30 minutes at -78 ${ }^{\circ} \mathrm{C}$, a solution of $t$-butylchloro(dimethyl)silane $10 \mathrm{~b}(0.39 \mathrm{~g}, 2.6 \mathrm{mmol})$ in $4 \mathrm{~mL}$ of THF was added. The reaction mixture was kept at $-78{ }^{\circ} \mathrm{C}$ during 30 minutes and was allowed to stir at room temperature overnight. After removal of the solvent under vacuum, the resulting crude was dissolved in toluene and was filtered off. After concentration under vacuum, 11b was obtained as a colorless uncrystallized compound $\left(0.65 \mathrm{~g}, 80 \%\right.$ yield). ${ }^{1} \mathrm{H}$ NMR $\left(300 \mathrm{MHz}, \mathrm{C}_{6} \mathrm{D}_{6}\right): \delta 0.19$ (d, $\left.{ }^{3} J_{\mathrm{PH}}=9.0 \mathrm{~Hz}, 6 \mathrm{H}, \mathrm{SiMe}_{2}\right) ; 0.90-5.50\left(\mathrm{br}, 3 \mathrm{H}, \mathrm{BH}_{3}\right) ; 0.90(\mathrm{~s}, 9 \mathrm{H}, t-\mathrm{Bu}) ; 7.01-7.06(\mathrm{~m}, 6 \mathrm{H}, \mathrm{Ph})$; 7.82-7.84 (m, 4H, Ph). ${ }^{13} \mathrm{C}$ NMR (75.4 MHz, $\left.\mathrm{C}_{6} \mathrm{D}_{6}\right): \delta-5.1\left(\mathrm{~d},{ }^{2} J_{\mathrm{PC}}=8.3 \mathrm{~Hz}, \mathrm{SiMe}_{2}\right) ; 19.9\left(\mathrm{~d}, J_{\mathrm{PC}}\right.$ $=9.3 \mathrm{~Hz}, t-\mathrm{Bu}) ; 27.4\left(\mathrm{~s}, \mathrm{CH}_{3}\right) ; 128.8\left(\mathrm{~d}, J_{\mathrm{PC}}=9.3 \mathrm{~Hz}, \mathrm{C}\right.$-aryl $) ; 130.5\left(\mathrm{~d}, J_{\mathrm{PC}}=2.3 \mathrm{~Hz}, \mathrm{C}\right.$-aryl $)$; $134.1\left(\mathrm{~d}, J_{\mathrm{PC}}=8.2 \mathrm{~Hz}, \mathrm{C}\right.$-aryl $) ; 135.3\left(\mathrm{~d}, J_{\mathrm{PC}}=18.6 \mathrm{~Hz}, \mathrm{C}\right.$-aryl). ${ }^{31} \mathrm{P} \mathrm{NMR}\left(121.4 \mathrm{MHz}, \mathrm{C}_{6} \mathrm{D}_{6}\right): \delta$ $-26.6\left(\mathrm{~d},{ }^{1} J_{\mathrm{PB}}=38 \mathrm{~Hz}\right) .{ }^{29} \mathrm{Si} \mathrm{NMR}\left(99.4 \mathrm{MHz}, \mathrm{C}_{6} \mathrm{D}_{6}\right): \delta+4.7\left(\mathrm{~d}, J_{\mathrm{SiP}}=44.3 \mathrm{~Hz}\right)$.

(R)-o-Anisyl(trimethylsilyl)phenylphosphine-borane (11c). To a solution of (S)-oanisylphenylphosphine-borane 7c $(0.086 \mathrm{~g}, 0.37 \mathrm{mmol}, 85 \%$ ee $)$ in $6 \mathrm{~mL}$ of THF under inert atmosphere, was added dropwise at $-78{ }^{\circ} \mathrm{C} n$-BuLi $(0.3 \mathrm{~mL}, 0.41 \mathrm{mmol}, 1.1$ equiv). After stirring 30 minutes at $-78{ }^{\circ} \mathrm{C}$, a solution of bromo(trimethyl)silane $10 \mathrm{c}(0.1 \mathrm{~mL}, 0.37 \mathrm{mmol})$ in $4 \mathrm{~mL}$ of THF was added. The reaction mixture was kept at $-78{ }^{\circ} \mathrm{C}$ during 30 minutes and was stirred at room temperature overnight. After removal under vacuum the solvent, the residue was dissolved in toluene and was filtered off. After concentration under vacuum, 11c was obtained as a colorless uncrystallized product $(0.10 \mathrm{~g}, 93 \%$ yield $) .[\alpha]_{\mathrm{D}}{ }^{25}=-57.1$ (c $\left.0.5, \mathrm{THF}\right) ;{ }^{1} \mathrm{H}$ NMR $(300$ $\left.\mathrm{MHz}, \mathrm{CDCl}_{3}\right): \delta 0.29\left(\mathrm{~d},{ }^{3} J=6.0 \mathrm{~Hz}, 9 \mathrm{H}, \mathrm{SiMe}_{3}\right) ; 0.50-1.50\left(\mathrm{br}, 3 \mathrm{H}, \mathrm{BH}_{3}\right) ; 3.82$ (s, OMe); 6.94$7.06(\mathrm{~m}, 2 \mathrm{H}, \mathrm{Ph}) ; 7.32-7.55(\mathrm{~m}, 6 \mathrm{H}, \mathrm{Ph}) ; 7.62-7.73(\mathrm{~m}, 1 \mathrm{H}, \mathrm{Ph}) .{ }^{13} \mathrm{C} \mathrm{NMR}\left(75.4 \mathrm{MHz}, \mathrm{C}_{6} \mathrm{D}_{6}\right): \delta-$ $1.6\left(\mathrm{~d},{ }^{2} J_{\mathrm{PC}}=9.4 \mathrm{~Hz}, \mathrm{SiMe}_{3}\right) ; 54.5(\mathrm{~s}, \mathrm{OMe}) ; 110.3\left(\mathrm{~d}, J_{\mathrm{PC}}=3.8 \mathrm{~Hz}, \mathrm{C}\right.$-aryl $) ; 116.8\left(\mathrm{~d}, J_{\mathrm{P}-\mathrm{C}}=44.4\right.$ $\mathrm{Hz}, \mathrm{C}$-aryl); $121.6\left(\mathrm{~d}, J_{\mathrm{PC}}=10.1 \mathrm{~Hz}, \mathrm{C}\right.$-aryl $) ; 128.4\left(\mathrm{~d}, J_{\mathrm{PC}}=10.1 \mathrm{~Hz}, \mathrm{C}\right.$-aryl $) ; 129.6\left(\mathrm{~d}, J_{\mathrm{PC}}=3.8\right.$ $\mathrm{Hz}, \mathrm{C}$-aryl); $129.9\left(\mathrm{~d}, J_{\mathrm{PC}}=44.4 \mathrm{~Hz}, \mathrm{C}\right.$-aryl); $132.5\left(\mathrm{~d}, J_{\mathrm{PC}}=3.8 \mathrm{~Hz}, \mathrm{C}\right.$-aryl); $132.7\left(\mathrm{~d}, J_{\mathrm{PC}}=10.1\right.$ $\mathrm{Hz}, \mathrm{C}$-aryl); $136.2\left(\mathrm{~d}, J_{\mathrm{PC}}=10.1 \mathrm{~Hz}, \mathrm{C}\right.$-aryl); $160.3\left(\mathrm{~s}, \mathrm{C}\right.$-aryl). ${ }^{31} \mathrm{P}$ NMR $\left(121.4 \mathrm{MHz}, \mathrm{CDCl}_{3}\right): \delta$ $-28.9\left(\mathrm{~d},{ }^{1} J_{\mathrm{PB}}=48.6 \mathrm{~Hz}\right) .{ }^{29} \mathrm{Si} \mathrm{NMR}\left(99.4 \mathrm{MHz}, \mathrm{CDCl}_{3}\right): \delta+6.4\left(\mathrm{~d},{ }^{1} J_{\mathrm{PSi}}=48.7 \mathrm{~Hz}\right)$.

(S)-Ferrocenyl(trimethylsilyl)phenylphosphine-borane (11d). To a solution of $(R)$ ferrocenylphenylphosphine-borane $7 \mathbf{d}(60 \mathrm{mg}, 0.19 \mathrm{mmol}, 87 \%$ ee $)$ in $2 \mathrm{~mL}$ of THF under inert 
atmosphere, was added dropwise at $-78{ }^{\circ} \mathrm{C} n$-BuLi $(0.09 \mathrm{~mL}, 0.21 \mathrm{mmol}, 1.1$ equiv). After stirring 30 minutes at $-78{ }^{\circ} \mathrm{C}$, a solution of bromo(trimethyl)silane $10 \mathrm{c}(0.03 \mathrm{~mL}, 0.23 \mathrm{mmol}, 1.2$ equiv) in $0.5 \mathrm{~mL}$ of THF was added and the reaction was stirred at room temperature overnight. After removal the solvent under vacuum, the residue was dissolved in toluene and was used without further purification. ${ }^{31} \mathrm{P}$ NMR $\left(121.4 \mathrm{MHz}\right.$, toluene $\left.\mathrm{D}_{8}\right): \delta-34.3\left(\mathrm{~d},{ }^{1} J_{\mathrm{PB}}=48.6 \mathrm{~Hz}\right)$.

4-Diphenylphosphinobutan-2-one (14a). ${ }^{46}$ To a solution of diphenyl(trimethylsilyl) phosphine $12(0.64 \mathrm{~g}, 2.47 \mathrm{mmol})$ in $10 \mathrm{~mL}$ of THF under inert atmosphere was added a solution of methylvinylketone 13a $(0.20 \mathrm{~mL}, 2.47 \mathrm{mmol})$ in $5 \mathrm{~mL}$ of THF at room temperature. After 16 hours stirring, the reaction mixture was concentrated under vacuum. After purification by chromatography on silica gel, 14a was obtained as a colorless uncrystallized compound $(0.32 \mathrm{~g}$, $51 \%$ yield). $R_{\mathrm{f}}=0.82$ (dichloromethane/pentane 80:20). ${ }^{1} \mathrm{H} \mathrm{NMR}\left(300 \mathrm{MHz}, \mathrm{CDCl}_{3}\right): \delta 2.13(\mathrm{~s}$, $3 \mathrm{H}, \mathrm{Me}) ; 2.33\left(\mathrm{~m}, 2 \mathrm{H}, \mathrm{CH}_{2}\right) ; 2.52\left(\mathrm{~m}, 2 \mathrm{H}, \mathrm{CH}_{2}\right)$; 7.32-7.40 (m, 6H, Ph); 7.41-7.50 (m, 4H, Ph). ${ }^{13} \mathrm{C} \mathrm{NMR}\left(75.4 \mathrm{MHz}, \mathrm{CDCl}_{3}\right): 21.1\left(\mathrm{~d}, J_{\mathrm{PC}}=11.2 \mathrm{~Hz}, \mathrm{CH}_{2}\right) ; 29.9(\mathrm{~s}, \mathrm{Me}) ; 39.8\left(\mathrm{~d}, J_{\mathrm{PC}}=17.7 \mathrm{~Hz}\right.$, C-P); $128.6\left(\mathrm{~d}, J_{\mathrm{PC}}=6.7 \mathrm{~Hz}, \mathrm{C}\right.$-aryl); 128.9 (s, C-aryl); 132.7 (d, $J_{\mathrm{PC}}=18.5 \mathrm{~Hz}, \mathrm{C}$-aryl); 138.2 (d, $J_{\mathrm{PC}}=12.5 \mathrm{~Hz}, \mathrm{C}$-aryl); $207.7\left(\mathrm{~d}, J_{\mathrm{PC}}=12.5 \mathrm{~Hz}, \mathrm{C}=\mathrm{O}\right) .{ }^{31} \mathrm{P} \mathrm{NMR}\left(121.4 \mathrm{MHz}, \mathrm{CDCl}_{3}\right): \delta-15.7$ (s). HRMS (ESI-Q-TOF) calcd for $\mathrm{C}_{16} \mathrm{H}_{17} \mathrm{OPNa}[\mathrm{M}+\mathrm{Na}]^{+}: 279.0909$, found 279.0911 .

5-Diphenylphosphinopentan-3-one (14b). ${ }^{47}$ To a solution of diphenyl(trimethylsilyl)phosphine $12(0.64 \mathrm{~g}, 2.47 \mathrm{mmol})$ in $10 \mathrm{~mL}$ of THF was added at room temperature under inert atmosphere a solution of ethylvinylketone $13 \mathbf{b}(0.17 \mathrm{~mL}, 2.47 \mathrm{mmol})$ in $5 \mathrm{~mL}$ of THF. After 16 hours stirring, the reaction mixture was concentrated under vacuum. After purification by chromatography on silica gel, $\mathbf{1 4 b}$ was obtained as colorless uncrystallized product $(0.39 \mathrm{~g}, 58 \%$ yield). $R_{\mathrm{f}}=0.76$ (dichloromethane/Pentane 80:20). ${ }^{1} \mathrm{H}$ NMR $\left(600 \mathrm{MHz}, \mathrm{CDCl}_{3}\right): \delta 1.02\left(\mathrm{t},{ }^{3} \mathrm{~J}=\right.$ $\left.7.4 \mathrm{~Hz}, 3 \mathrm{H}, \mathrm{CH}_{3}\right) ; 2.30\left(\mathrm{~m}, \mathrm{CH}_{2}\right) ; 2.38\left(\mathrm{q},{ }^{3} J=7.4 \mathrm{~Hz}, 2 \mathrm{H}, \mathrm{CH}_{2}\right) ; 2.49$ (td, $J=7.7 \mathrm{~Hz}, J=8.4 \mathrm{~Hz}$, $\left.2 \mathrm{H}, \mathrm{CH}_{2}\right)$; 7.33-7.39 (m, 6H, Ph); 7.40-7.50 (m, 4H, Ph). $\left.{ }^{13} \mathrm{C} \mathrm{NMR} \mathrm{(75.4} \mathrm{MHz,} \mathrm{CDCl}_{3}\right): \delta 7.8(\mathrm{~s}$, $\left.\mathrm{CH}_{3}\right) ; 21.4\left(\mathrm{~d},{ }^{1} J_{\mathrm{PC}}=11.2 \mathrm{~Hz}, \mathrm{CH}_{2} \mathrm{P}\right) ; 35.9\left(\mathrm{~s}, \mathrm{CH}_{2}\right) ; 38.4\left(\mathrm{~d},{ }^{2} J_{\mathrm{PC}}=17.7 \mathrm{~Hz}, \mathrm{CH}_{2}\right) ; 128.5\left(\mathrm{~d}, J_{\mathrm{PC}}\right.$ $=6.7 \mathrm{~Hz}, \mathrm{C}$-aryl); 128.8 (s, C-aryl); 132.7 (d, $J_{\mathrm{PC}}=18.5 \mathrm{~Hz}, \mathrm{C}$-aryl); 138.2 (d, $J_{\mathrm{PC}}=12.5 \mathrm{~Hz}, \mathrm{C}-$ aryl); $210.4\left(\mathrm{~d}, \mathrm{~J}_{\mathrm{P}-\mathrm{C}}=12.5 \mathrm{~Hz}, \mathrm{C}=\mathrm{O}\right) .{ }^{31} \mathrm{P} \mathrm{NMR}\left(121.4 \mathrm{MHz}, \mathrm{CDCl}_{3}\right): \delta-15.4$ (s). HRMS (ESI-QTOF) calcd for $\mathrm{C}_{17} \mathrm{H}_{19} \mathrm{OPNa}[\mathrm{M}+\mathrm{Na}]^{+}: 293.0923$, found: 293.0916.

(R)-4-[(Boranato)-o-anisylphenylphosphino]butan-2-one (14c). To a solution of $(R)$-oanisyl(trimethylsilyl)phenylphosphine-borane 11c $(0.05 \mathrm{~g}, 0.17 \mathrm{mmol})$ in $5 \mathrm{~mL}$ of THF under inert atmosphere was added at $-78{ }^{\circ} \mathrm{C}$ a solution of methylvinylketone $13 \mathbf{a}(14 \mu \mathrm{L}, 0.17 \mathrm{mmol})$ in $5 \mathrm{~mL}$ of THF. After 16 hours stirring, the reaction mixture was concentrated under vacuum and the residue was purified by chromatography on silica gel to afford $\mathbf{1 4 c}$ as a colorless uncrystallized compound $\left(0.04 \mathrm{~g}, 72 \%\right.$ yield). $R_{\mathrm{f}}=0.86$ (dichloromethane). ${ }^{1} \mathrm{H}$ NMR (300 MHz, $\mathrm{CDCl}_{3}$ ): $\delta$ 0.50-1.70 (br, 3H, BH $) ; 2.11\left(\mathrm{~s}, \mathrm{CH}_{3}\right) ; 2.53\left(\mathrm{~m}, 2 \mathrm{H}, \mathrm{CH}_{2}\right) ; 2.80\left(\mathrm{~m}, 2 \mathrm{H}, \mathrm{CH}_{2} \mathrm{P}\right) ; 3.69$ $\left(\mathrm{s}, 3 \mathrm{H}, \mathrm{OCH}_{3}\right) ; 6.90(\mathrm{dd}, J=3.0 \mathrm{~Hz}, J=6.0 \mathrm{~Hz}, 1 \mathrm{H}, \mathrm{Ph}) ; 7.10(\mathrm{t}, J=6.0 \mathrm{~Hz}, 1 \mathrm{H}, \mathrm{Ph}) ; 7.39-7.45$ $(\mathrm{m}, 3 \mathrm{H}, \mathrm{Ph}) ; 7.53(\mathrm{t}, J=6.0 \mathrm{~Hz}, 1 \mathrm{H}, \mathrm{Ph}) ; 7.68(\mathrm{dd}, J=3.0 \mathrm{~Hz}, J=6.0 \mathrm{~Hz}, 2 \mathrm{H}, \mathrm{Ph}) ; 7.91$ (dd, $J=$ $6.0 \mathrm{~Hz}, 1 \mathrm{H}, \mathrm{Ph}) .{ }^{13} \mathrm{C} \mathrm{NMR}\left(75.4 \mathrm{MHz}, \mathrm{CDCl}_{3}\right): \delta 17.7\left(\mathrm{~d}, J_{\mathrm{PC}}=41.5 \mathrm{~Hz}, \mathrm{CH}_{2} \mathrm{P}\right) ; 29.8\left(\mathrm{~s}, \mathrm{CH}_{3}\right)$; $37.3\left(\mathrm{~d}, J_{\mathrm{PC}}=2.4 \mathrm{~Hz}, \mathrm{CH}_{2}\right) ; 55.4\left(\mathrm{~s}, \mathrm{OCH}_{3}\right) ; 111.1\left(\mathrm{~d}, J_{\mathrm{PC}}=4.9 \mathrm{~Hz}, \mathrm{C}\right.$-aryl $) ; 115.6\left(\mathrm{~d}, J_{\mathrm{PC}}=51.0\right.$ $\mathrm{Hz}, \mathrm{C}$-aryl); $121.2\left(\mathrm{~d}, J_{\mathrm{PC}}=12.2 \mathrm{~Hz}, \mathrm{C}\right.$-aryl $) ; 128.4\left(\mathrm{~d}, J_{\mathrm{PC}}=12.2 \mathrm{~Hz}, \mathrm{C}\right.$-aryl $) ; 129.8\left(\mathrm{~d}, J_{\mathrm{PC}}=\right.$ 
$57.1 \mathrm{~Hz}, \mathrm{C}$-aryl); $130.6\left(\mathrm{~d}, J_{\mathrm{PC}}=2.4 \mathrm{~Hz}, \mathrm{CH}_{2}\right) ; 131.6\left(\mathrm{~d}, J_{\mathrm{PC}}=12.2 \mathrm{~Hz}, \mathrm{C}\right.$-aryl $) ; 134.0\left(\mathrm{~d}, J_{\mathrm{PC}}=\right.$ $\left.2.4 \mathrm{~Hz}, \mathrm{CH}_{2}\right) ; 136.4\left(\mathrm{~d}, J_{\mathrm{PC}}=12.1 \mathrm{~Hz}, \mathrm{C}\right.$-aryl $) ; 206.7\left(\mathrm{~d}, J_{\mathrm{PC}}=13.4 \mathrm{~Hz}, \mathrm{C}=\mathrm{O}\right) .{ }^{31} \mathrm{P}$ NMR $(121.4$ $\left.\mathrm{MHz}, \mathrm{CDCl}_{3}\right): \delta+15.7\left(\mathrm{~d}, J_{\mathrm{PB}}=76 \mathrm{~Hz}\right.$ ). HRMS (ESI-Q-TOF) calcd for $\mathrm{C}_{17} \mathrm{H}_{22} \mathrm{O}_{2} \mathrm{PBNa}$ $[\mathrm{M}+\mathrm{Na}]^{+}: 323.1342$, found: 323.1335 . The enantiomeric excess $82 \%$ was determined by HPLC on Chiralcel OK column using a mixture hexane/ $i$-propanol 80:20 as eluent, flow $1 \mathrm{~mL} / \mathrm{min}, \lambda=$ $230 \mathrm{~nm}, \mathrm{~T}=40^{\circ} \mathrm{C}$. The retention times for the enantiomers were $\mathrm{t}_{1}=15.3$ and $\mathrm{t}_{2}=30.5$ minutes, respectively. ${ }^{48}$

(S)-5-[(Boranato)ferrocenylphenylphosphino]pentan-3-one (14d). To a solution of $(R)$ ferrocenyl(trimethylsilyl)phenylphosphine-borane $11 \mathrm{~d}(0.07 \mathrm{~g}, 0.19 \mathrm{mmol}, 33 \%$ ee) in $2 \mathrm{~mL}$ of THF under inert atmosphere, was added at $-78{ }^{\circ} \mathrm{C}$ a solution of ethylvinylketone $13 \mathbf{b}(38.6 \mu \mathrm{L}$, $0.39 \mathrm{mmol}$ ) in $0.5 \mathrm{~mL}$ of THF. The reaction mixture was then allowed to warm up at room temperature. After 16 hours stirring, the reaction mixture was concentrated under vacuum and the residue was purified by chromatography on silica gel to afford $\mathbf{1 4 d}$ as an orange uncrystallized compound $\left(0.07 \mathrm{~g}, 95 \%\right.$ yield). $R_{\mathrm{f}}=0.74$ (dichloromethane/ethyl acetate 97:3); $[\alpha]_{\mathrm{D}}{ }^{25}=-10.1\left(\mathrm{c} 1.4, \mathrm{CHCl}_{3}\right), 33 \%$ ee uncorrected. ${ }^{1} \mathrm{H}$ NMR $\left(300 \mathrm{MHz}, \mathrm{C}_{6} \mathrm{D}_{6}\right): \delta 0.70(\mathrm{t}, J=7.20$ $\left.\mathrm{Hz}, 3 \mathrm{H}, \mathrm{CH}_{3}\right) ; 1.40-1.90\left(\mathrm{~m}, 3 \mathrm{H}, \mathrm{BH}_{3}\right) ; 1.54\left(\mathrm{AB}, J=17.9 \mathrm{~Hz}, J=7.2 \mathrm{~Hz}, 1 \mathrm{H}, \mathrm{CH}_{2}\right) ; 1.62(\mathrm{AB}, J$ $\left.=17.9 \mathrm{~Hz}, J=7.2 \mathrm{~Hz}, 1 \mathrm{H}, \mathrm{CH}_{2}\right) ; 2.05-2.20\left(\mathrm{~m}, 1 \mathrm{H}, \mathrm{CH}_{2}\right) ; 2.25-2.36\left(\mathrm{~m}, 2 \mathrm{H}, \mathrm{CH}_{2}\right) ; 2.45-2.60(\mathrm{~m}$, 1H, $\mathrm{CH}_{2}$ ); 3.92-3.95 (m, 1H, Fc); 3.95 (s, 5H, Fc); 3.97-4.00 (m, 1H, Fc); 4.14-4.17 (m, 1H, Fc); 4.36-4.38 (m, 1H, Fc); 6.93-6.99 (m, 3H, Ph); 7.69-7.76 (m, 2H, Ph). ${ }^{13} \mathrm{C}$ NMR $(75.4 \mathrm{MHz}$, $\left.\mathrm{C}_{6} \mathrm{D}_{6}\right): \delta 7.6\left(\mathrm{~s}, \mathrm{CH}_{3}\right) ; 21.5\left(\mathrm{~d}, J_{\mathrm{PC}}=40.6 \mathrm{~Hz}, \mathrm{CH}_{2} \mathrm{P}\right) ; 35.2\left(\mathrm{~s}, \mathrm{CH}_{2}\right) ; 35.6\left(\mathrm{~d}, J_{\mathrm{PC}}=2.1 \mathrm{~Hz}, \mathrm{CH}_{2}\right)$; $70.0\left(\mathrm{~s}, \mathrm{CH}_{\mathrm{Fc}}\right) ; 70.5\left(\mathrm{~d}, J_{\mathrm{PC}}=64.9 \mathrm{~Hz}, \mathrm{Fc}\right) ; 71.2\left(\mathrm{~d}, J_{\mathrm{PC}}=7.5 \mathrm{~Hz}, \mathrm{Fc}\right) ; 71.8\left(\mathrm{~d}, J_{\mathrm{PC}}=8.8 \mathrm{~Hz}, \mathrm{Fc}\right)$; $71.8\left(\mathrm{~d}, J_{\mathrm{PC}}=7.9 \mathrm{~Hz}, \mathrm{Fc}\right) ; 71.9\left(\mathrm{~d}, J_{\mathrm{PC}}=9.8 \mathrm{~Hz}, \mathrm{Fc}\right) ; 128.6\left(\mathrm{~d}, J_{\mathrm{PC}}=9.7 \mathrm{~Hz}, \mathrm{Ph}\right) ; 130.7\left(\mathrm{~d}, J_{\mathrm{PC}}=\right.$ $54.0 \mathrm{~Hz}, \mathrm{Ph}) ; 131.0\left(\mathrm{~d}, J_{\mathrm{PC}}=2.4 \mathrm{~Hz}, \mathrm{Ph}\right) ; 132.4\left(\mathrm{~d}, J_{\mathrm{PC}}=9.1 \mathrm{~Hz}, \mathrm{Ph}\right) ; 207.3\left(\mathrm{~d}, J_{\mathrm{PC}}=12.0 \mathrm{~Hz}\right.$, $\mathrm{C}=\mathrm{O}) .{ }^{31} \mathrm{P}$ NMR (121.4 MHz, $\left.\mathrm{C}_{6} \mathrm{D}_{6}\right): \delta+26.0 \quad(\mathrm{~m})$. HRMS (ESI-Q-TOF) calcd for $\mathrm{C}_{21} \mathrm{H}_{26} \mathrm{BFeOPNa}[\mathrm{M}+\mathrm{Na}]^{+}:$415.10601, found: 415.10756. FTIR (neat): 3056, 2976, 2937, 2373, 1714, 1436, 1413, 1172, 1107, 1063, 1026, 742. The enantiomeric excess $33 \%$ was determined by HPLC on Chiralcel OD-H column using a mixture hexane/i-propanol 97:3 as eluent, flow 0.7 $\mathrm{mL} / \mathrm{min}$., $\lambda=210 \mathrm{~nm}, \mathrm{~T}=40{ }^{\circ} \mathrm{C}$. The retention times for the enantiomers were $\mathrm{t}_{1}=17.07$ and $\mathrm{t}_{2}$ $=20.25$ minutes, respectively. ${ }^{48}$

3-(Trimethylsilyloxy)but-2-enyl-diphenylphosphine-borane (15a). To a solution of diphenyl(trimethylsilyl)phosphine-borane $11 \mathrm{a}(0.65 \mathrm{~g}, 3.33 \mathrm{mmol})$ in $10 \mathrm{~mL}$ of THF under inert atmosphere was added a solution of methylvinylketone $13 \mathbf{a}(0.30 \mathrm{~mL}, 3.33 \mathrm{mmol})$ in $5 \mathrm{~mL}$ of THF at room temperature. After 16 hours stirring, the reaction mixture was concentrated under vacuum and the residue was purified by chromatography on silica gel to afford 15a as a mixture of isomers in 2:1 ratio and colorless uncrystallized compound ( $0.45 \mathrm{~g}, 40 \%$ yield). $R_{\mathrm{f}}=0.79$ (dichloromethane/pentane 80:20). ${ }^{1} \mathrm{H} \mathrm{NMR}\left(300 \mathrm{MHz}, \mathrm{CDCl}_{3}\right.$ ): 0.50-1.50 (br, 3H, $\mathrm{BH}_{3}$ ); Major isomer, $\delta 0.20\left(\mathrm{~s}, 6 \mathrm{H}, \mathrm{SiMe}_{3}\right) ; 1.76\left(\mathrm{dd}, J=1.0 \mathrm{~Hz}, J=3.9 \mathrm{~Hz}, 1.3 \mathrm{H}, \mathrm{CH}_{3}\right) ; 3.06(\mathrm{dd}, J=7.3 \mathrm{~Hz}$, $\left.J=12.0 \mathrm{~Hz}, 1.3 \mathrm{H}, \mathrm{CH}_{2}\right) ; 4.50(\mathrm{q}, J=7.2 \mathrm{~Hz}, 0.7 \mathrm{H}, \mathrm{CH}=\mathrm{CO})$; [Minor isomer, $0.10(\mathrm{~s}, 3 \mathrm{H}$, $\left.\mathrm{SiMe}_{3}\right) ; 1.60\left(\mathrm{dd}, J=0.6 \mathrm{~Hz}, J=3.2 \mathrm{~Hz}, 0.7 \mathrm{H}, \mathrm{CH}_{3}\right) ; 2.99(\mathrm{dd}, J=8.1 \mathrm{~Hz}, J=11.9 \mathrm{~Hz}, 0.7 \mathrm{H}$, $\left.\left.\mathrm{CH}_{2}\right) ; 4.60(\mathrm{q}, J=6.3 \mathrm{~Hz}, 0.3 \mathrm{H}, \mathrm{CH}=\mathrm{CO})\right]$; 7.43-7.49 (m, 6H, Ph); 7.67-7.74 (m, 4H, Ph). ${ }^{13} \mathrm{C}$ 
NMR (75.4 MHz, $\left.\mathrm{C}_{6} \mathrm{D}_{6}\right)$ : major isomer, $\delta 1.10\left(\mathrm{~s}, \mathrm{SiMe}_{3}\right) ; 22.4\left(\mathrm{~d}, J_{\mathrm{PC}}=2.3 \mathrm{~Hz}, \mathrm{CH}_{3}\right) ; 23.4(\mathrm{~d}$, $\left.J_{\mathrm{PC}}=38.5 \mathrm{~Hz}, \mathrm{CH}_{2}\right) ; 97.2\left(\mathrm{~d}, J_{\mathrm{PC}}=4.6 \mathrm{~Hz}, \mathrm{CH}=\mathrm{C}-\mathrm{O}\right) ; 128.6\left(\mathrm{~d}, J_{\mathrm{PC}}=10.4 \mathrm{~Hz}, \mathrm{C}-\operatorname{aryl}\right) ; 129.7(\mathrm{~d}$, $J_{\mathrm{PC}}=54.3 \mathrm{~Hz}, \mathrm{C}$-aryl); $130.9\left(\mathrm{~d}, J_{\mathrm{PC}}=5.2 \mathrm{~Hz}, \mathrm{C}\right.$-aryl); $132.3\left(\mathrm{~d}, J_{\mathrm{PC}}=2.3 \mathrm{~Hz}, \mathrm{C}\right.$-aryl); $150.3(\mathrm{~d}$, $\left.J_{\mathrm{PC}}=11.0 \mathrm{~Hz}, \mathrm{C}-\mathrm{OSiMe}_{3}\right)$. Minor isomer, $\delta 1.06\left(\mathrm{~s}, \mathrm{SiMe}_{3}\right) ; 18.1\left(\mathrm{~d}, J_{\mathrm{PC}}=2.3 \mathrm{~Hz}, \mathrm{CH}_{3}\right) ; 25.9(\mathrm{~d}$, $\left.J=38.5 \mathrm{~Hz}, \mathrm{CH}_{2}\right) ; 97.3\left(\mathrm{~d}, J_{\mathrm{PC}}=4.6 \mathrm{~Hz}, \mathrm{CH}=\mathrm{C}-\mathrm{O}\right) ; 129.1\left(\mathrm{~d}, J_{\mathrm{PC}}=10.4 \mathrm{~Hz}, \mathrm{C}-\operatorname{aryl}\right) ; 129.8(\mathrm{~d}$, $J_{\mathrm{PC}}=54.3 \mathrm{~Hz}, \mathrm{C}$-aryl $) ; 131.2\left(\mathrm{~d}, J_{\mathrm{PC}}=5.2 \mathrm{~Hz}, \mathrm{C}\right.$-aryl $) ; 132.5\left(\mathrm{~d}, J_{\mathrm{PC}}=2.3 \mathrm{~Hz}, \mathrm{C}\right.$-aryl); $152.1(\mathrm{~d}$, $\left.\left.J_{\mathrm{PC}}=11.0 \mathrm{~Hz}, \mathrm{C}-\mathrm{OSiMe}\right)_{3}\right) .{ }^{31} \mathrm{P} \mathrm{NMR}\left(121.4 \mathrm{MHz}, \mathrm{CDCl}_{3}\right): \delta+16.8(\mathrm{~d}, J=58.3 \mathrm{~Hz}) .{ }^{29} \mathrm{Si} \mathrm{NMR}$ $\left(99.4 \mathrm{MHz}, \mathrm{C}_{6} \mathrm{D}_{6}\right): \delta+17.3$. MS (ESI) m/z (relative intensity \%): 293 [M-SiMe 3 ; 100], 279[M$\left.\mathrm{BH}_{3}-\mathrm{SiMe}_{3} ; 60\right]$.

3-(Trimethylsilyloxy)pent-2-enyl-diphenylphosphine-borane (15b). To a solution of diphenyl(trimethylsilyl)phosphine-borane $11 \mathrm{a}(0.51 \mathrm{~g}, 1.85 \mathrm{mmol})$ in $5 \mathrm{~mL}$ of THF was added a solution of ethylvinylketone $\mathbf{1 3 b}(0.13 \mathrm{~mL}, 1.85 \mathrm{mmol})$ in $5 \mathrm{~mL}$ of $\mathrm{THF}$ at room temperature under inert atmosphere. After 16 hours stirring, the reaction mixture was concentrated under vacuum and the residue was purified by chromatography on silica gel to afford $\mathbf{1 5 b}$ as a mixture of isomers in 2:1 ratio and colorless uncrystallized compound $\left(0.31 \mathrm{~g}, 48 \%\right.$ yield). $R_{\mathrm{f}}=0.73$ (dichloromethane/pentane 80:20). ${ }^{1} \mathrm{H}$ NMR (300 MHz, $\mathrm{CDCl}_{3}$ ): 0.50-1.50 (br, 3H, $\mathrm{BH}_{3}$ ); Major isomer, $0.20\left(\mathrm{~s}, 6 \mathrm{H}, \mathrm{SiMe}_{3}\right) ; 0.93\left(\mathrm{t}, J=6 \mathrm{~Hz}, 2 \mathrm{H}, \mathrm{CH}_{3}\right) ; 1.98\left(\mathrm{~m}, 1.3 \mathrm{H}, \mathrm{CH}_{2}\right) ; 3.02(\mathrm{dm}, J=6$ $\left.\mathrm{Hz}, 1.3 \mathrm{H}, \mathrm{CH}_{2} \mathrm{P}\right) ; 4.56(\mathrm{~m}, 0.7 \mathrm{H}, \mathrm{CH}=\mathrm{C})$; [Minor isomer, $\delta 0.15\left(\mathrm{~s}, 3 \mathrm{H}, \mathrm{SiMe}_{3}\right) ; 0.87(\mathrm{t}, J=6 \mathrm{~Hz}$, $\left.\left.1 \mathrm{H}, \mathrm{CH}_{3}\right) ; 1.99\left(\mathrm{~m}, 0.7 \mathrm{H}, \mathrm{CH}_{2}\right) ; 2.98\left(\mathrm{dm}, J=6 \mathrm{~Hz}, 0.7 \mathrm{H}, \mathrm{CH}_{2}-\mathrm{P}\right) ; 4.58(\mathrm{~m}, 0.3 \mathrm{H}, \mathrm{CH}=\mathrm{C})\right] ; 7.41-$ $7.48(\mathrm{~m}, 6 \mathrm{H}, \mathrm{Ph}) ; 7.67-7.74(\mathrm{~m}, 4 \mathrm{H}, \mathrm{Ph}) .{ }^{13} \mathrm{C} \mathrm{NMR}\left(75.4 \mathrm{MHz}, \mathrm{CDCl}_{3}\right)$ : Major isomer, $\delta 0.4$ $\left(\mathrm{SiMe}_{3}\right) ; 10.7\left(\mathrm{~s}, \mathrm{CH}_{3}\right) ; 22.5\left(\mathrm{~d}, J_{\mathrm{PC}}=37.7 \mathrm{~Hz}, \mathrm{CH}_{2}-\mathrm{P}\right) ; 28.4\left(\mathrm{~d}, J_{\mathrm{PC}}=2.3 \mathrm{~Hz}, \mathrm{CH}_{2}\right) ; 95.2(\mathrm{~d}, J=$ $4.5 \mathrm{~Hz}, \mathrm{CH}=\mathrm{C}) ; 127.8\left(\mathrm{~d}, J_{\mathrm{PC}}=19.6 \mathrm{~Hz}, \mathrm{C}-\operatorname{aryl}\right) ; 128.8\left(\mathrm{~d}, J_{\mathrm{PC}}=53.6 \mathrm{~Hz}, \mathrm{C}\right.$-aryl $) ; 130.1\left(\mathrm{~d}, J_{\mathrm{PC}}=\right.$ $3.0 \mathrm{~Hz}, \mathrm{C}$-aryl); $131.5\left(\mathrm{~d}, J_{\mathrm{PC}}=9.0 \mathrm{~Hz}, \mathrm{C}\right.$-aryl); 154.7 (d, $\left.J_{\mathrm{PC}}=11.3 \mathrm{~Hz}, \mathrm{C}-\mathrm{OSiMe}_{3}\right)$; Minor isomer, $\delta 0.2\left(\mathrm{SiMe}_{3}\right) ; 10.3\left(\mathrm{~s}, \mathrm{CH}_{3}\right) ; 22.6\left(\mathrm{~d}, J_{\mathrm{PC}}=37.7 \mathrm{~Hz}, \mathrm{CH}_{2}\right) ; 24.7\left(\mathrm{~d}, J_{\mathrm{PC}}=2.3 \mathrm{~Hz}, \mathrm{CH}_{2}-\mathrm{P}\right)$; $94.8(\mathrm{~d}, J=4.5 \mathrm{~Hz}, \mathrm{CH}=\mathrm{C}) ; 128.0\left(\mathrm{~d}, J_{\mathrm{PC}}=19.6 \mathrm{~Hz}, \mathrm{C}\right.$-aryl $) ; 130.3\left(\mathrm{~d}, J_{\mathrm{PC}}=3.0 \mathrm{~Hz}, \mathrm{C}\right.$-aryl); $131.2\left(\mathrm{~d}, J_{\mathrm{PC}}=9.1 \mathrm{~Hz}, \mathrm{C}\right.$-aryl $) ; 155.9\left(\mathrm{~d}, J_{\mathrm{PC}}=12.1 \mathrm{~Hz}, \mathrm{C}-\mathrm{OSiMe}_{3}\right) .{ }^{31} \mathrm{P}$ NMR $(121.4 \mathrm{MHz}$, $\left.\mathrm{CDCl}_{3}\right): \delta+16.7(\mathrm{sl}) .{ }^{29} \mathrm{Si} \mathrm{NMR}\left(99.4 \mathrm{MHz}, \mathrm{CDCl}_{3}\right): \delta+17.3$. MS (ESI) $\mathrm{m} / \mathrm{z}$ (relative intensity \%): 307 [M-SiMe $;$; 100], $293\left[\mathrm{M}-\mathrm{BH}_{3}-\mathrm{SiMe}_{3} ; 60\right]$.

( \pm )-[3-(Trimethylsilyloxy)cyclohex-2-enyl]diphenylphosphine-borane (15c). To a solution of diphenyl(trimethylsilyl)phosphine-borane 11a $(0.32 \mathrm{~g}, 1.19 \mathrm{mmol})$ in $5 \mathrm{~mL}$ of THF under inert atmosphere was added at room temperature a solution of cyclohex-2-enone 13c $(0.13 \mathrm{~mL}, 1.31$ mmol) in $5 \mathrm{~mL}$ of THF. After 16 hours stirring, the reaction mixture was concentrated under vacuum and the residue was purified by chromatography on silica gel to afford $15 \mathbf{c}$ as a colorless uncrystallized compound ( $0.43 \mathrm{~g}, 84 \%$ yield). $R_{\mathrm{f}}=0.83$ (dichloromethane/ethyl acetate 95:5). ${ }^{1} \mathrm{H}$ NMR (300 MHz, $\left.\mathrm{C}_{6} \mathrm{D}_{6}\right): \delta 0.17$ (s, 9H, SiMe $)$; 0.90-2.20 (br, 3H, BH $) ; 1.30\left(\mathrm{~m}, 2 \mathrm{H}, \mathrm{CH}_{2}\right) ; 1.55$ (m, 2H, $\left.\mathrm{CH}_{2}\right) ; 1.85\left(\mathrm{~m}, 2 \mathrm{H}, \mathrm{CH}_{2}\right) ; 3.26(\mathrm{~m}, 1 \mathrm{H}, \mathrm{CH}-\mathrm{P}) ; 4.64$ (dm, J= $\left.8 \mathrm{~Hz}, 1 \mathrm{H}, \mathrm{CH}=\mathrm{CO}\right)$; 6.987.09 (m, 6H, Ph); 7.67-7.87 (m, 4H, Ph). $\left.{ }^{13} \mathrm{C} \mathrm{NMR} \mathrm{(75.4} \mathrm{MHz,} \mathrm{C}_{6} \mathrm{D}_{6}\right): \delta 0.2\left(\mathrm{~s}, \mathrm{SiMe}_{3}\right) ; 22.3(\mathrm{~d}$, $\left.J_{\mathrm{PC}}=9.8 \mathrm{~Hz}, \mathrm{CH}_{2}\right) ; 23.1\left(\mathrm{~s}, \mathrm{CH}_{2}\right) ; 29.7\left(\mathrm{~d}, J_{\mathrm{PC}}=2.6 \mathrm{~Hz}, \mathrm{CH}_{2}\right) ; 32.7\left(\mathrm{~d}, J_{\mathrm{PC}}=36.2 \mathrm{~Hz}, \mathrm{CH}-\mathrm{P}\right)$; $99.6\left(\mathrm{~d}, J_{\mathrm{PC}}=2.3 \mathrm{~Hz}, \mathrm{CH}=\mathrm{CO}\right) ; 128.5\left(\mathrm{~d}, J_{\mathrm{PC}}=3.0 \mathrm{~Hz}, \mathrm{C}\right.$-aryl); $128.7\left(\mathrm{~d}, J_{\mathrm{PC}}=3.0 \mathrm{~Hz}, \mathrm{C}\right.$-aryl $)$; $130.7\left(\mathrm{~d}, J_{\mathrm{PC}}=2.3 \mathrm{~Hz}, \mathrm{C}\right.$-aryl $) ; 131.0\left(\mathrm{~d}, J_{\mathrm{PC}}=2.3 \mathrm{~Hz}, \mathrm{C}\right.$-aryl $) ; 132.6\left(\mathrm{~d}, J_{\mathrm{PC}}=8.3 \mathrm{~Hz}, \mathrm{C}\right.$-aryl $)$; 
$133.3\left(\mathrm{~d}, J_{\mathrm{PC}}=8.3 \mathrm{~Hz}, \mathrm{C}\right.$-aryl $) ; 154.5\left(\mathrm{~d}, J_{\mathrm{PC}}=9.8 \mathrm{~Hz}, \mathrm{C}-\mathrm{OSSiMe}_{3}\right) .{ }^{31} \mathrm{P} \mathrm{NMR}(121.4 \mathrm{MHz}$, $\left.\mathrm{C}_{6} \mathrm{D}_{6}\right): \delta+22.6$ (sl). ${ }^{29} \mathrm{Si} \mathrm{NMR}\left(99.4 \mathrm{MHz}, \mathrm{C}_{6} \mathrm{D}_{6}\right): \delta+16.8$. HRMS (ESI-Q-TOF) calcd for $\mathrm{C}_{21} \mathrm{H}_{30}$ BOPSiNa [M+Na] $]^{+}$391.1792, found: 391.1768.

$( \pm)-3-[t$-Butyl(dimethyl)silyloxy] cyclohex-2-enyl(diphenyl)phosphine-borane $\quad(15 \mathrm{~d})$. To a solution of diphenyl[ $t$-butyl(dimethyl)silyl]phosphine-borane $11 \mathrm{~b}(0.88 \mathrm{~g}, 2.8 \mathrm{mmol})$ in $6 \mathrm{~mL}$ of THF was added to a solution of cyclohex-2-enone $13 \mathrm{c}(0.28 \mathrm{~mL}, 2.9 \mathrm{mmol})$ at room temperature and under inert atmosphere. After $16 \mathrm{~h}$ of stirring, the reaction mixture was concentrated under vacuum and the residue was purified by chromatography on silica gel (toluene/pentane 70:30) to afford 15d as a colorless uncrystallized compound ( $0.88 \mathrm{~g}, 77 \%$ yield). ${ }^{1} \mathrm{H}$ NMR $(300 \mathrm{MHz}$, $\mathrm{C}_{6} \mathrm{D}_{6}$ ): $\delta 0.03$ (s, 6H, SiMe $)$; 0.15 (s, 9H, Sit-Bu); 0.90-2.30 (br, 3H, $\left.\mathrm{BH}_{3}\right) ; 1.20\left(\mathrm{~m}, 2 \mathrm{H}, \mathrm{CH}_{2}\right.$ ); $1.55\left(\mathrm{~m}, 2 \mathrm{H}, \mathrm{CH}_{2}\right) ; 1.80\left(\mathrm{~m}, 2 \mathrm{H}, \mathrm{CH}_{2}\right) ; 3.13(\mathrm{~m}, 1 \mathrm{H}, \mathrm{CH}-\mathrm{P}) ; 4.67$ (dd, $1 \mathrm{H}, J=8.4 \mathrm{~Hz}, J=1.3 \mathrm{~Hz}$, $\mathrm{CH}=\mathrm{CO}) ; 6.80-7.10(\mathrm{~m}, 6 \mathrm{H}, \mathrm{Ph}) ; 7.50-7.80(\mathrm{~m}, 4 \mathrm{H}, \mathrm{Ph}) .{ }^{13} \mathrm{C} \mathrm{NMR}\left(75.4 \mathrm{MHz}, \mathrm{C}_{6} \mathrm{D}_{6}\right): \delta 0.2(\mathrm{~s}$, $\left.\mathrm{SiMe}_{2}\right) ; 1.0$ (s, Sit-Bu); 22.5 (d, $\left.J_{\mathrm{PC}}=9.8 \mathrm{~Hz}, \mathrm{CH}_{2}\right) ; 23.4\left(\mathrm{~s}, \mathrm{CH}_{2}\right) ; 29.9\left(\mathrm{~d}, J_{\mathrm{PC}}=2.3 \mathrm{~Hz}, \mathrm{CH}_{2}\right)$; $32.9\left(\mathrm{~d}, J_{\mathrm{PC}}=36.8 \mathrm{~Hz}, \mathrm{CH}-\mathrm{P}\right) ; 99.8\left(\mathrm{~d}, J_{\mathrm{PC}}=2.3 \mathrm{~Hz}, \mathrm{CH}=\mathrm{CO}\right) ; 129.2\left(\mathrm{~d}, J_{\mathrm{PC}}=10.4 \mathrm{~Hz}, \mathrm{C}\right.$-aryl); $131.6\left(\mathrm{~d}, J_{\mathrm{PC}}=2.2 \mathrm{~Hz}, \mathrm{C}\right.$-aryl); $132.9\left(\mathrm{~d}, J_{\mathrm{PC}}=9.9 \mathrm{~Hz}, \mathrm{C}\right.$-aryl); $136.6(\mathrm{~s}, \mathrm{C}-\operatorname{aryl}) ; 137.2(\mathrm{~s}, \mathrm{C}-$ $\operatorname{aryl}) ; 137.3\left(\mathrm{~d}, J_{\mathrm{PC}}=3.8 \mathrm{~Hz}, \mathrm{C}\right.$-aryl); $154.7\left(\mathrm{~d}, J_{\mathrm{PC}}=10.4 \mathrm{~Hz}, \mathrm{C}-\mathrm{OSiMe}_{3}\right) .{ }^{31} \mathrm{P} \mathrm{NMR}(121.4$ $\left.\mathrm{MHz}, \mathrm{C}_{6} \mathrm{D}_{6}\right): \delta+21.3(\mathrm{sl})$.

(S)-[3-(Trimethylsilyloxy)cyclohex-2-enyl]ferrocenylphenylphosphine-borane (15e). To a solution of $(R)$-ferrocenylphenylphosphine-borane $7 \mathbf{d}(60 \mathrm{mg}, 0.19 \mathrm{mmol}, 1$ equiv., $87 \%$ ee) in 2 $\mathrm{mL}$ of toluene at $-90{ }^{\circ} \mathrm{C}$ was added a solution of $n$-BuLi $(0.09 \mathrm{~mL}, 0.21 \mathrm{mmol}, 1.1$ equiv). The temperature was stirred until $-80{ }^{\circ} \mathrm{C}$ and after 30 minutes, bromo(trimethyl)silane 10c $(30 \mu \mathrm{L}$, $0.23 \mathrm{mmol}, 1.2$ equiv) was added at $-90^{\circ} \mathrm{C}$. The reaction mixture was stirred during 3 hours from $-90{ }^{\circ} \mathrm{C}$ up to $-60{ }^{\circ} \mathrm{C}$, and a solution of cyclohexenone $13 \mathrm{c}(37 \mu \mathrm{L}, 0.39 \mathrm{mmol})$ in $0.5 \mathrm{~mL}$ of THF was added. The reaction mixture was then allowed to warm up at room temperature. After 16 hours stirring, the reaction mixture was concentrated under vacuum and the residue was purified by chromatography on silica gel to afford 15e as a yellowish uncrystallized mixture of two diastereosiomers in 1:1 ratio $\left(0.07 \mathrm{~g}, 75 \%\right.$ yield). $R_{\mathrm{f}}=0.89$ (dichloromethane/ethyl acetate 98:2); $[\alpha]_{\mathrm{D}}{ }^{25}=-68.0\left(\mathrm{c} 2, \mathrm{CHCl}_{3}\right) 87 \%$ ee uncorrected. ${ }^{1} \mathrm{H}$ NMR $\left(300 \mathrm{MHz}, \mathrm{C}_{6} \mathrm{D}_{6}\right): \delta 0.00(\mathrm{~s}, 4.5 \mathrm{H}$, $\mathrm{SiMe}_{3}$ ); 0.12 (s, 4.5H, $\mathrm{SiMe}_{3}$ ); 1.05-1.39 (m, 2H, $\left.\mathrm{CH}_{2}\right) ; 0.90-2.20$ (m, 3H, BH $) ; 1.41-1.57$ (m, $\left.1 \mathrm{H}, \mathrm{CH}_{2}\right) ; 1.57-1.70\left(\mathrm{~m}, 1 \mathrm{H}, \mathrm{CH}_{2}\right) ; 1.71-1.97\left(\mathrm{~m}, 2 \mathrm{H}, \mathrm{CH}_{2}\right) ; 2.79-2.85(\mathrm{~m}, 1 \mathrm{H}, \mathrm{CHP}) ; 3.77$ (s, 2.5H, Fc); 3.81 (s, 2.5H, Fc); 4.00 (sl, 3H, Fc); 4.58 (d, J=7.81 Hz, 0.5H, Fc); 4.67 (s, 0.5H, $\mathrm{CH}=) ; 4.74(\mathrm{~s}, 0.5 \mathrm{H}, \mathrm{CH}=) ; 4.78(\mathrm{~d}, J=7.8 \mathrm{~Hz}, 0.5 \mathrm{H}, \mathrm{Fc}) ; 6.85-7.05(\mathrm{~m}, 3 \mathrm{H}, \mathrm{Ph}) ; 7.65-7.90(\mathrm{~m}$, 2H, Ph). ${ }^{13} \mathrm{C}$ NMR (75.4 MHz, $\left.\mathrm{C}_{6} \mathrm{D}_{6}\right): \delta 0.4\left(\mathrm{~s}, \mathrm{SiMe}_{3}\right) ; 0.6\left(\mathrm{~s}, \mathrm{SiMe}_{3}\right) ; 22.4\left(\mathrm{~d}, J_{\mathrm{PC}}=4.4 \mathrm{~Hz}\right.$, $\left.\mathrm{CH}_{2}\right) ; 22.6\left(\mathrm{~d}, J_{\mathrm{PC}}=4.8 \mathrm{~Hz}, \mathrm{CH}_{2}\right) ; 23.7\left(\mathrm{~s}, \mathrm{CH}_{2}\right) ; 23.9\left(\mathrm{~s}, \mathrm{CH}_{2}\right) ; 30.0\left(\mathrm{~m}, \mathrm{CH}_{2}\right) ; 35.8\left(\mathrm{~d}, J_{\mathrm{PC}}=\right.$ $18.6 \mathrm{~Hz}, \mathrm{CHP}) ; 36.8\left(\mathrm{~d}, J_{\mathrm{PC}}=18.7 \mathrm{~Hz}, \mathrm{CHP}\right) ; 69.6\left(\mathrm{~d}, J_{\mathrm{PC}}=61.1 \mathrm{~Hz}, \mathrm{Fc}\right) ; 69.6\left(\mathrm{~d}, J_{\mathrm{PC}}=59.6 \mathrm{~Hz}\right.$, $\mathrm{Fc}) ; 70.1(\mathrm{~s}, \mathrm{Fc}) ; 70.2(\mathrm{~s}, \mathrm{Fc}) ; 71.0(\mathrm{~m}, \mathrm{Fc}) ; 71.1\left(\mathrm{~d}, J_{\mathrm{PC}}=8.1 \mathrm{~Hz}, \mathrm{Fc}\right) ; 71.2\left(\mathrm{~d}, J_{\mathrm{PC}}=8.3 \mathrm{~Hz}, \mathrm{Fc}\right)$; $71.8\left(\mathrm{~d}, J_{\mathrm{PC}}=5.9 \mathrm{~Hz}, \mathrm{Fc}\right) ; 72.1\left(\mathrm{~d}, J_{\mathrm{PC}}=6.1 \mathrm{~Hz}, \mathrm{Fc}\right) ; 74.5\left(\mathrm{~d}, J_{\mathrm{PC}}=14.7 \mathrm{~Hz}, \mathrm{Fc}\right) ; 75.1\left(\mathrm{~d}, J_{\mathrm{PC}}=\right.$ $14.4 \mathrm{~Hz}, \mathrm{Fc}) ; 100.3\left(\mathrm{~d}, J_{\mathrm{PC}}=1.5 \mathrm{~Hz}, \mathrm{CH}=\right) ; 100.8\left(\mathrm{~d}, J_{\mathrm{PC}}=2.7 \mathrm{~Hz}, \mathrm{CH}=\right) ; 128.3\left(\mathrm{~d}, J_{\mathrm{PC}}=10.3\right.$ $\mathrm{Hz}, \mathrm{Ph}) ; 128.4\left(\mathrm{~d}, J_{\mathrm{PC}}=9.5 \mathrm{~Hz}, \mathrm{CH}_{\mathrm{Ph}}\right) ; 129.6\left(\mathrm{~d}, J_{\mathrm{PC}}=53.3 \mathrm{~Hz}, \mathrm{Ph}\right) ; 130.1\left(\mathrm{~d}, J_{\mathrm{PC}}=51.7 \mathrm{~Hz}, \mathrm{Ph}\right)$; $131.1\left(\mathrm{~d}, J_{\mathrm{PC}}=2.3 \mathrm{~Hz}, \mathrm{Ph}\right) ; 131.2\left(\mathrm{~d}, J_{\mathrm{PC}}=2.2 \mathrm{~Hz}, \mathrm{Ph}\right) ; 133.0\left(\mathrm{~d}, J_{\mathrm{PC}}=8.3 \mathrm{~Hz}, \mathrm{Ph}\right) ; 133.4\left(\mathrm{~d}, J_{\mathrm{PC}}\right.$ 
$=8.1 \mathrm{~Hz}, \mathrm{Ph}) ; 154.2\left(\mathrm{~d}, J_{\mathrm{PC}}=2.7 \mathrm{~Hz},=\mathrm{CH}-\mathrm{O}\right) ; 154.4\left(\mathrm{~d}, J_{\mathrm{PC}}=3.2 \mathrm{~Hz},=\mathrm{CH}-\mathrm{O}\right) .{ }^{31} \mathrm{P}$ NMR $(121.4$ $\mathrm{MHz}, \mathrm{CDCl}_{3}$ ): $\delta+33.7(\mathrm{~m})$. HRMS (ESI-Q-TOF) calcd for $\mathrm{C}_{25} \mathrm{H}_{34} \mathrm{BFeOPSiNa}[\mathrm{M}+\mathrm{Na}]^{+}$: 499.14561, found 499.14140. FTIR (neat): 3057, 3005, 2938, 2385, 2348, 1657, 1369, 1252, $1198,1174,904,844,748$. The enantiomeric (87\% ee) and diasteromeric purities (1:1) was determined by HPLC on Chiralcel OK column using a mixture hexane/ $i$-propanol 97:3 as eluent, flow $1 \mathrm{~mL} / \mathrm{min}$., $\lambda=254 \mathrm{~nm}$., $\mathrm{T}=30{ }^{\circ} \mathrm{C}$. The retention times for the stereoisomers were $\mathrm{t}_{1}=$ $11.8, \mathrm{t}_{1^{\prime}}=15.2, \mathrm{t}_{2}=13.7$ and $\mathrm{t}_{2^{\prime}}=25.4$ minutes, respectively. ${ }^{48}$

( \pm )-[3-(Trimethylsilyloxy)cyclohex-2-enyl]diphenylphosphine (16). To a solution of [3(trimethylsilyloxy)cyclohex-2-enyl](diphenyl)phosphine-borane $15 \mathrm{c}(1.47 \mathrm{~g}, 4.0 \mathrm{mmol})$ in 10 $\mathrm{mL}$ of degassed toluene was added at room temperature under stirring, a solution of DABCO $(0.8 \mathrm{~g}, 8.0 \mathrm{mmol})$ in $10 \mathrm{~mL}$ of toluene. After 16 hours stirring, the reaction mixture was concentrated under vacuum and the residue was purified by chromatography on silica gel to afford 16 as a colorless uncrystallized compound (1.41 g, $100 \%$ yield). ${ }^{1} \mathrm{H}$ NMR (300 MHz, $\mathrm{CDCl}_{3}$ ): $\delta 0.03\left(\mathrm{~s}, 9 \mathrm{H}, \mathrm{SiMe}_{3}\right) ; 1.70\left(\mathrm{~m}, 2 \mathrm{H}, \mathrm{CH}_{2}\right) ; 1.95\left(\mathrm{~m}, 2 \mathrm{H}, \mathrm{CH}_{2}\right) ; 2.35\left(\mathrm{~m}, 2 \mathrm{H}, \mathrm{CH}_{2}\right) ; 3.09$ (m, 1H, CH-P); 4.56 (m, 1H, CH=CO); 7.24-7.33 (m, 6H, Ph); 7.40-7.53 (m, 4H, Ph). ${ }^{13} \mathrm{C} \mathrm{NMR}$ $\left(75.4 \mathrm{MHz}, \mathrm{CDCl}_{3}\right): \delta 0.2\left(\mathrm{~s}, \mathrm{SiMe}_{3}\right) ; 21.6\left(\mathrm{~d}, J_{\mathrm{PC}}=9.8 \mathrm{~Hz}, \mathrm{CH}_{2}\right) ; 25.6\left(\mathrm{~d}, J_{\mathrm{PC}}=17.3 \mathrm{~Hz}, \mathrm{CH}_{2}\right)$; $29.8\left(\mathrm{~d}, J_{\mathrm{PC}}=2.2 \mathrm{~Hz}, \mathrm{CH}_{2}\right) ; 33.1\left(\mathrm{~d}, J_{\mathrm{PC}}=21.1 \mathrm{~Hz}, \mathrm{CH}_{2}-\mathrm{P}\right) ; 103.6\left(\mathrm{~d}, J_{\mathrm{PC}}=7.5 \mathrm{~Hz}, \mathrm{CH}=\mathrm{CO}\right)$; $128.6\left(\mathrm{~d}, J_{\mathrm{PC}}=3.0 \mathrm{~Hz}, \mathrm{C}\right.$-aryl $) ; 130.3\left(\mathrm{~d}, J_{\mathrm{PC}}=2.3 \mathrm{~Hz}, \mathrm{C}\right.$-aryl $) ; 133.6\left(\mathrm{~d}, J_{\mathrm{PC}}=8.3 \mathrm{~Hz}, \mathrm{C}\right.$-aryl $)$; $137.9\left(\mathrm{~d}, J_{\mathrm{PC}}=17.4 \mathrm{~Hz}, \mathrm{C}\right.$-aryl); $152.2\left(\mathrm{~d}, J_{\mathrm{PC}}=8.2 \mathrm{~Hz}, \mathrm{CO}\right) .{ }^{31} \mathrm{P} \mathrm{NMR}\left(121.4 \mathrm{MHz}, \mathrm{CDCl}_{3}\right): \delta-$ 6.2 (s). ${ }^{29} \mathrm{Si} \mathrm{NMR}\left(99.4 \mathrm{MHz}, \mathrm{C}_{6} \mathrm{D}_{6}\right): \delta+16.2$. MS (ESI) m/z (relative intensity \%): 282 [M$\left.\mathrm{SiMe}_{3}, 100\right]$.

Methyl 3-[(boranato)diphenylphosphino]propenoate (18). ${ }^{49}$ To a solution of diphenyl(trimethyl)silylphosphine-borane $11 \mathrm{a}(0.32 \mathrm{~g}, 1.19 \mathrm{mmol})$ in $10 \mathrm{~mL}$ of THF was added a solution of methyl propiolate $\mathbf{1 3 d}(0.11 \mathrm{~mL}, 1.2 \mathrm{mmol})$ in $4 \mathrm{~mL}$ of THF under inert atmosphere at room temperature. After 16 hours stirring, the reaction mixture was concentrated under vacuum and the residue was purified by chromatography on silica gel to afford $\mathbf{1 8}$ as a colorless uncrystallized compound. ( $0.19 \mathrm{~g}, 56 \%$ yield). $R_{\mathrm{f}}=0,76$ (toluene/pentane 80:20). ${ }^{1} \mathrm{H}$ NMR (300 $\left.\mathrm{MHz}, \mathrm{C}_{6} \mathrm{D}_{6}\right): \delta$ 0.90-2.20 (br, 3H, $\left.\mathrm{BH}_{3}\right) ; 3.25(\mathrm{~s}, 3 \mathrm{H}, \mathrm{OMe}) ; 6.79(\mathrm{dd}, 1 \mathrm{H}, J=16.7 \mathrm{~Hz}, J=16.6$ $\mathrm{Hz}, \mathrm{CH}) ; 7.60(\mathrm{dd}, 1 \mathrm{H}, J=10 \mathrm{~Hz}, J=16.7 \mathrm{~Hz}, \mathrm{CH}) ; 6.89-7.01(\mathrm{~m}, 6 \mathrm{H}, \mathrm{Ph}) ; 7.46-7.53(\mathrm{~m}, 4 \mathrm{H}$, $\mathrm{Ph}) .{ }^{13} \mathrm{C}$ NMR (75.4 MHz, $\left.\mathrm{C}_{6} \mathrm{D}_{6}\right): \delta 50.2(\mathrm{OMe}) ; 127.7\left(\mathrm{~d}, J_{\mathrm{PC}}=9.9 \mathrm{~Hz}, \mathrm{C}\right.$-aryl); $130.1\left(\mathrm{~d}, J_{\mathrm{PC}}=\right.$ $2.2 \mathrm{~Hz}, \mathrm{C}$-aryl); $131.5\left(\mathrm{~d}, J_{\mathrm{PC}}=9.9 \mathrm{~Hz}, \mathrm{C}\right.$-aryl); $135.5\left(\mathrm{~d}, J_{\mathrm{PC}}=45.2 \mathrm{~Hz}, \mathrm{PCH}=\right) ; 136.0\left(\mathrm{~d}, J_{\mathrm{PC}}=\right.$ $3.9 \mathrm{~Hz}, \mathrm{C}=\mathrm{C}) ; 163.0(\mathrm{~d}, J=19.5 \mathrm{~Hz}, \mathrm{CO}) .{ }^{31} \mathrm{P} \mathrm{NMR}\left(121.4 \mathrm{MHz}, \mathrm{C}_{6} \mathrm{D}_{6}\right): \delta+14.9\left(\mathrm{dl},{ }^{1} J_{\mathrm{PB}}=62\right.$ $\mathrm{Hz}$ ). HRMS (ESI-Q-TOF) calcd for $\mathrm{C}_{16} \mathrm{H}_{18} \mathrm{BO}_{2} \mathrm{PNa}[\mathrm{M}+\mathrm{Na}]^{+}: 307.1032$, found: 307.1023 .

( \pm )-1-Phenyl-3,4-bis[(boranato)diphenylphosphino]pyrrolidine-2,5-dione (19a).

To a solution of diphenylphosphine-borane 7a $\left(\mathrm{R}^{1}, \mathrm{R}^{2}=\mathrm{Ph}\right)(182 \mathrm{mg}, 0.91 \mathrm{mmol}, 2.2$ equiv) in 4 $\mathrm{mL}$ of toluene was added at $-78{ }^{\circ} \mathrm{C}$ a solution of $n$-BuLi $(0.36 \mathrm{~mL}, 0.91 \mathrm{mmol}, 2.2$ equiv). After 30 minutes stirring, bromo(trimethyl)silane $10 \mathrm{c}(0.13 \mathrm{~mL}, 0.99 \mathrm{mmol}, 2.4$ equiv) was added. After stirring 2 hours at $-78{ }^{\circ} \mathrm{C}$, a solution of 2,3-dichloromaleimide $13 \mathbf{e}(0.10 \mathrm{~g}, 0.41 \mathrm{mmol})$ in $0.5 \mathrm{~mL}$ of THF was added and the reaction mixture was then allowed to warm up at room temperature. After 16 hours stirring, the reaction mixture was concentrated under vacuum and 
the residue was purified by chromatography on silica gel to afford the compound 19a as a white solid $\left(0.20 \mathrm{~g}, 86 \%\right.$ yield). $R_{\mathrm{f}}=0.75$ (dichloromethane/pentane 80:20). ${ }^{1} \mathrm{H}$ NMR $(300 \mathrm{MHz}$, $\left.\mathrm{CDCl}_{3}\right): \delta 0.50-1.50\left(\mathrm{br}, 6 \mathrm{H}, \mathrm{BH}_{3}\right) ; 4.32-4.41(\mathrm{AB}, J=11.1 \mathrm{~Hz}, 2 \mathrm{H}, \mathrm{CH}) ; 6.86-6.92(\mathrm{~m}, 2 \mathrm{H}, \mathrm{Ph}-$ $\mathrm{N}) ; 7.29-7.35(\mathrm{~m}, 3 \mathrm{H}, \mathrm{Ph}-\mathrm{N}) ; 7.40-7.62(\mathrm{~m}, 12 \mathrm{H}, \mathrm{Ph}) ; 7.75-7.86(\mathrm{~m}, 8 \mathrm{H}, \mathrm{Ph}) .{ }^{13} \mathrm{C} \mathrm{NMR}(75.4$ $\left.\mathrm{MHz}, \mathrm{CDCl}_{3}\right): \delta 40.8\left(\mathrm{~d}, J_{\mathrm{PC}}=25.7 \mathrm{~Hz}, \mathrm{CHP}\right) ; 125.1(\mathrm{~d}, J=55.9 \mathrm{~Hz}, \mathrm{C}-\operatorname{aryl}) ; 126.6$ (s, C-aryl); 128.9 (s, C-aryl); 129.1 (s, C-aryl), 129.1 (d, $J_{\mathrm{PC}}=9.1 \mathrm{~Hz}, \mathrm{C}$-aryl); 129.3 (d, $J_{\mathrm{PC}}=9.1 \mathrm{~Hz}, \mathrm{C}-$ $\operatorname{aryl}) ; 131.1$ (s, C-aryl, Ph-N); $132.4\left(\mathrm{~d}, J_{\mathrm{PC}}=12.8 \mathrm{~Hz}, \mathrm{C}\right.$-aryl); $133.2\left(\mathrm{~d}, J_{\mathrm{PC}}=9.1 \mathrm{~Hz}, \mathrm{C}\right.$-aryl); $133.4\left(\mathrm{~d}, J_{\mathrm{PC}}=9.1 \mathrm{~Hz}, \mathrm{C}\right.$-aryl); $170.6(\mathrm{~s}, \mathrm{C}=\mathrm{O}) .{ }^{31} \mathrm{P} \mathrm{NMR}\left(121.4 \mathrm{MHz}, \mathrm{CDCl}_{3}\right): \delta+29.6(\mathrm{sl})$. HRMS (ESI-Q-TOF) calcd for $\mathrm{C}_{34} \mathrm{H}_{33} \mathrm{~B}_{2} \mathrm{P}_{2} \mathrm{NO}_{2} \mathrm{Na}[\mathrm{M}+\mathrm{Na}]^{+}$: 594.2070, found: 594.2091. FTIR (neat): $3058,2950,2392,2349,1713,1497,1436,1378,1192,1105,1060,1028,737,689$. An additional fraction was isolated as a colorless uncrystallized product that corresponds to the compound 20a (27 mg, $11 \%$ yield).

(士)-1-Benzyl-3,4-bis[(boranato)diphenylphosphino]pyrrolidine-2,5-dione (19b).

To a solution of 2,3-dibromomaleimide $\mathbf{1 3 f}(0.142 \mathrm{~g}, 0.41 \mathrm{mmol})$ in $10 \mathrm{~mL}$ of THF under inert atmosphere was added at $-78{ }^{\circ} \mathrm{C}$ a solution of diphenyl(trimethylsilyl)phosphine-borane 11a $(0.215 \mathrm{~g}, 0.83 \mathrm{mmol})$ in $10 \mathrm{~mL}$ of THF. After 16 hours stirring, the reaction mixture was concentrated under vacuum and the residue was purified by chromatography on silica gel to afford the compound $\mathbf{1 9 b}$ as a colorless uncrystallized compound $\left(0.12 \mathrm{~g}, 50 \%\right.$ yield). $R_{\mathrm{f}}=0.73$ (dichloromethane/pentane 80:20). ${ }^{1} \mathrm{H}$ NMR (300 MHz, $\mathrm{CDCl}_{3}$ ): $\delta 0.50-1.50$ (br, 6H, $\mathrm{BH}_{3}$ ); 4.26$4.32(\mathrm{AB}, J=12.0 \mathrm{~Hz}, 2 \mathrm{H}, \mathrm{CH}) ; 4.34$ (s, 2H, $\left.\mathrm{CH}_{2} \mathrm{Ph}\right) ; 7.09-7.99$ (m, 25H, Ph). ${ }^{13} \mathrm{C}$ NMR $(75.4$ $\left.\mathrm{MHz}, \mathrm{CDCl}_{3}\right): \delta 40.6\left(\mathrm{~d}, J_{\mathrm{PC}}=27.2 \mathrm{~Hz}, \mathrm{C}-\mathrm{P}\right) ; 43.6\left(\mathrm{~s}, \mathrm{CH}_{2} \mathrm{Ph}\right) ; 125.2(\mathrm{~d}, J=55.8 \mathrm{~Hz}, \mathrm{C}$-aryl); 127.9 (s, C-aryl); 128.5 (s, C-aryl); $128.8\left(\mathrm{~d}, J_{\mathrm{PC}}=10.6 \mathrm{~Hz}, \mathrm{C}\right.$-aryl); 129.1 (d, $J_{\mathrm{PC}}=10.6 \mathrm{~Hz}, \mathrm{C}-$ $\operatorname{aryl}) ; 129.6$ (s, C-aryl); 132.2 (d, $J_{\mathrm{PC}}=12.1 \mathrm{~Hz}, \mathrm{C}$-aryl); 133.0 (d, $J_{\mathrm{PC}}=9.8 \mathrm{~Hz}, \mathrm{C}$-aryl); 133.3 (d, $J_{\mathrm{PC}}=9.8 \mathrm{~Hz}, \mathrm{C}$-aryl); $134.4\left(\mathrm{~s}, \mathrm{C}\right.$-aryl); $171.6(\mathrm{~s}, \mathrm{C}=\mathrm{O}) .{ }^{31} \mathrm{P}$ NMR $\left(121.4 \mathrm{MHz}, \mathrm{CDCl}_{3}\right): \delta+28.9$ (sl). HRMS (ESI-Q-TOF) calcd for $\mathrm{C}_{35} \mathrm{H}_{35} \mathrm{~B}_{2} \mathrm{P}_{2} \mathrm{NO}_{2} \mathrm{Na}$ [M+Na] $]^{+}$: 608.2227, found: 608.2248.

\section{1-Phenyl-3-[(boranato)diphenylphosphino]-4-(diphenylphosphino)pyrrole-2,5-dione (20a).}

To a solution of 2,3-dichloromaleimide $13 \mathrm{e}(0.10 \mathrm{~g}, 0.41 \mathrm{mmol})$ in $5 \mathrm{~mL}$ of diethylether under inert atmosphere was added at $0{ }^{\circ} \mathrm{C}$ a solution of diphenyl(trimethylsilyl)phosphine-borane 11a $(0.215 \mathrm{~g}, 0.83 \mathrm{mmol})$ in $5 \mathrm{~mL}$ of diethylether. After 16 hours stirring the reaction mixture was concentrated under vacuum and the residue was purified by chromatography on silica gel to afford the compound 20a as a colorless uncrystallized compound ( $0.08 \mathrm{~g}, 35 \%$ yield). $R_{\mathrm{f}}=0.20$ (pentane/ethyl acetate 90:10). ${ }^{1} \mathrm{H}$ NMR $\left(300 \mathrm{MHz}, \mathrm{CDCl}_{3}\right): \delta 1.00-2.00\left(\mathrm{br}, 3 \mathrm{H}, \mathrm{BH}_{3}\right) ; 7.23-7.26$ (m, 2H, Ph); 7.30-7.39 (m, 15H, Ph); 7.44 (td, $J=3.0 \mathrm{~Hz}, J=6.0 \mathrm{~Hz}, 3 \mathrm{H}, \mathrm{Ph}) ; 7.53$ (td, $J=3.0$ $\mathrm{Hz}, J=6.0 \mathrm{~Hz}, 2 \mathrm{H}, \mathrm{Ph}) ; 7.81-7.86(\mathrm{~m}, 3 \mathrm{H}, \mathrm{Ph}) .{ }^{13} \mathrm{C} \mathrm{NMR}\left(125.8 \mathrm{MHz}, \mathrm{CDCl}_{3}\right): \delta 125.8(\mathrm{~s}, \mathrm{C}-$ $\operatorname{aryl}) ; 127.3\left(\mathrm{~d}, J_{\mathrm{PC}}=48.5 \mathrm{~Hz}, \mathrm{C}\right.$-aryl); 127.9 (s, C-aryl); $128.5\left(\mathrm{~d}, J_{\mathrm{PC}}=7.3 \mathrm{~Hz}, \mathrm{C}\right.$-aryl); 128.6 (d, $J_{\mathrm{PC}}=3.6 \mathrm{~Hz}, \mathrm{C}$-aryl); $128.9\left(\mathrm{~d}, J_{\mathrm{PC}}=5.0 \mathrm{~Hz}, \mathrm{C}\right.$-aryl); $129.0(\mathrm{~s}, \mathrm{C}$-aryl); 129.4 (s, C-aryl); 131.2 $(\mathrm{s}, \mathrm{C}-\operatorname{aryl}) ; 131.9\left(\mathrm{~d}, J_{\mathrm{PC}}=2.7 \mathrm{~Hz}, \mathrm{C}\right.$-aryl $) ; 132.9\left(\mathrm{~d}, J_{\mathrm{PC}}=9.7 \mathrm{~Hz}, \mathrm{C}-\operatorname{aryl}\right) ; 133.4\left(\mathrm{~d}, J_{\mathrm{PC}}=9.7 \mathrm{~Hz}\right.$, C-aryl); 133.8 (s, C-aryl); 134.0 (s, C-aryl); 134.1 (d, $J_{\mathrm{PC}}=9.7 \mathrm{~Hz}, \mathrm{C}$-aryl); 155.1 (dd, $J_{\mathrm{PC}}=49.8$ $\mathrm{Hz}, J=4.8 \mathrm{~Hz}, \mathrm{C}-\mathrm{P}) ; 166.2(\mathrm{~m}, \mathrm{C}=\mathrm{O}) .{ }^{31} \mathrm{P} \mathrm{NMR}\left(121.4 \mathrm{MHz}, \mathrm{CDCl}_{3}\right): \delta+13.3(\mathrm{sl}),-28.3$ (s). HRMS (ESI-Q-TOF) calcd for $\mathrm{C}_{34} \mathrm{H}_{28} \mathrm{BP}_{2} \mathrm{NO}_{2} \mathrm{Na}[\mathrm{M}+\mathrm{Na}]^{+}$: 578.1580, found: 578.1573. FTIR 
(neat): 3054, 2925, 2855, 2409, 2363, 1712, 1500, 1483, 1434, 1373, 1100, 1052, 1027, 738, 688.

1-Benzyl-3-[(boranato)diphenylphosphino]-4-(diphenylphosphino)pyrrole-2,5-dione (20b). To a solution of diphenylphosphine borane $7 \mathbf{a}\left(\mathrm{R}^{1}, \mathrm{R}^{2}=\mathrm{Ph}\right)(182 \mathrm{mg}, 0.91 \mathrm{mmol}, 2.2$ equiv. $)$ in 4 $\mathrm{mL}$ of toluene was added at $-78^{\circ} \mathrm{C}$ a solution of $n$-BuLi $(0.36 \mathrm{~mL}, 0.91 \mathrm{mmol}, 2.2$ equiv.). After 30 minutes, bromo(trimethyl)silane $10 \mathrm{c}(0.13 \mathrm{~mL}, 0.99 \mathrm{mmol}, 2.4$ equiv. $)$ was then added. After 2 hours stirring at $-78^{\circ} \mathrm{C}$, a solution of 2,3-dibromomaleimide $\mathbf{1 3 f}(0.142 \mathrm{~g}, 0.41 \mathrm{mmol})$ in 0.5 $\mathrm{mL}$ of THF was added. The reaction mixture was then allowed to warm up at room temperature. After 16 hours stirring, the reaction mixture was concentrated under vacuum and the residue was purified by chromatography on silica gel to afford the compound $20 \mathrm{~b}$ as a solid $(0.225 \mathrm{~g}, 96 \%$ yield). $R_{\mathrm{f}}=0.75$ (dichloromethane/pentane 80:20). $\mathrm{Mp}=208^{\circ} \mathrm{C} ;{ }^{1} \mathrm{H} \mathrm{NMR}\left(300 \mathrm{MHz}, \mathrm{CDCl}_{3}\right) ; \delta$ 0.50-1.50 (br, $\left.3 \mathrm{H}, \mathrm{BH}_{3}\right) ; 4.56\left(\mathrm{~s}, 2 \mathrm{H}, \mathrm{CH}_{2} \mathrm{Ph}\right) ; 7.19-7.84$ (m, 25H, Ph). ${ }^{13} \mathrm{C}$ NMR $(75.4 \mathrm{MHz}$, $\mathrm{CDCl}_{3}$ ): $\delta 42.5\left(\mathrm{~s}, \mathrm{CH}_{2} \mathrm{Ph}\right) ; 125.3(\mathrm{~d}, J=55.8 \mathrm{~Hz}, \mathrm{C}-\mathrm{P}) ; 126.9$ (d, $\left.J=57.6 \mathrm{~Hz}, \mathrm{C}-\mathrm{P}\right) ; 127.8$ (s, C$\operatorname{aryl}) ; 128.5$ (s, C-aryl); 128.9 (d, $J_{\mathrm{P}-\mathrm{C}}=10.6 \mathrm{~Hz}, \mathrm{C}$-aryl); 129.1 (d, $J_{\mathrm{P}-\mathrm{C}}=10.6 \mathrm{~Hz}, \mathrm{C}$-aryl); 129.6 (s, C-aryl); $131.7(\mathrm{~d}, J=1.6 \mathrm{~Hz}) ; 132.3\left(\mathrm{~d}, J_{\mathrm{PC}}=12.1 \mathrm{~Hz}, \mathrm{C}-\operatorname{aryl}\right) ; 133.0\left(\mathrm{~d}, J_{\mathrm{PC}}=9.8 \mathrm{~Hz}, \mathrm{C}-\right.$ $\operatorname{aryl}) ; 133.3\left(\mathrm{~d}, J_{\mathrm{PC}}=9.8 \mathrm{~Hz}, \mathrm{C}\right.$-aryl $) ; 134.4(\mathrm{~s}, \mathrm{C}$-aryl $) ; 167.1\left(\mathrm{~d}, J_{\mathrm{PC}}=8.1 \mathrm{~Hz}, \mathrm{C}=\mathrm{O}\right) .{ }^{31} \mathrm{P}$ NMR $\left(121.4 \mathrm{MHz}, \mathrm{CDCl}_{3}\right.$ ): $\delta+12.8$ (sl); -28.2 (s). HRMS (ESI-Q-TOF) calcd for $\mathrm{C}_{35} \mathrm{H}_{30} \mathrm{BP}_{2} \mathrm{NO}_{2} \mathrm{Na}$ $[\mathrm{M}+\mathrm{Na}]^{+}:$592.1737; found: 592.1727. FTIR (neat): 3057, 2929, 2394, 2349, 1705, 1434, 1389, $1337,1102,1052,737,690$.

2-Chloro-3-(diphenylphosphino)quinoxaline (21). ${ }^{50}$ To a solution of diphenyl phosphineborane $7 \mathbf{a}\left(\mathrm{R}^{1}, \mathrm{R}^{2}=\mathrm{Ph}\right)(182 \mathrm{mg}, 0.91 \mathrm{mmol}, 2.2$ equiv. $)$ in $4 \mathrm{~mL}$ of toluene was added at $-78^{\circ} \mathrm{C}$ a solution of $n$-BuLi ( $0.36 \mathrm{~mL}, 0.91 \mathrm{mmol}, 2,2$ equiv.). After 30 minutes, bromo(trimethyl)silane 10c $\left(0.13 \mathrm{~mL}, 0.99 \mathrm{mmol}, 2.4\right.$ equiv.) was added. After 2 hours stirring at $-78^{\circ} \mathrm{C}$, a solution of 2,3-dichloroquinoxaline $13 \mathrm{~g}(0.08 \mathrm{~g}, 0.41 \mathrm{mmol})$ in $0.5 \mathrm{~mL}$ of THF was added. The reaction mixture was then allowed to warm up at room temperature. After 16 hours stirring, the reaction mixture was concentrated under vacuum and the residue was purified by chromatography on silica gel to afford the compound 21 as a white solid $(0.15 \mathrm{~g}, 46 \%$ yield $) . R_{\mathrm{f}}=0.77$ (dichloromethane/pentane 80:20). $\mathrm{Mp}=128^{\circ} \mathrm{C} ;{ }^{1} \mathrm{H}$ NMR $\left(300.1 \mathrm{MHz}, \mathrm{CDCl}_{3}\right): \delta$ 7.38-7.49 (10H, H-arom); 7.69-7.86 (m, 2H); $7.92(\mathrm{dd}, J=4.8 \mathrm{~Hz}, J=0.9 \mathrm{~Hz}, 1 \mathrm{H}) ; 8.15$ (dd, $J=4.8 \mathrm{~Hz}, J$ $=0.9 \mathrm{~Hz}, 1 \mathrm{H}) .{ }^{13} \mathrm{C} \mathrm{NMR}\left(75.4 \mathrm{MHz}, \mathrm{CDCl}_{3}\right): \delta 128.1(\mathrm{~s}, \mathrm{C}$-aryl $) ; 128.6\left(\mathrm{~d}, J_{\mathrm{PC}}=7.6 \mathrm{~Hz}, \mathrm{C}\right.$-aryl $)$; $128.8\left(\mathrm{~d}, J_{\mathrm{PC}}=10.6 \mathrm{~Hz}\right.$ C-aryl); $129.6\left(\mathrm{~d}, J_{\mathrm{PC}}=7.6 \mathrm{~Hz}, \mathrm{C}\right.$-aryl); 129.9 (s, C-aryl); 131.0 (s, C$\operatorname{aryl}$ ); 131.3 (s, C-aryl); 133.7 (d, $J_{\mathrm{PC}}=10.6 \mathrm{~Hz}, \mathrm{C}$-aryl); 133.9 (d, $J_{\mathrm{PC}}=7.6 \mathrm{~Hz}, \mathrm{C}$-aryl); 134.7 (d, $J_{\mathrm{PC}}=20.4 \mathrm{~Hz}, \mathrm{C}$-aryl); $141.0\left(\mathrm{~s}, \mathrm{C}\right.$-aryl); $141.6\left(\mathrm{~s}, \mathrm{C}\right.$-aryl); $150.6\left(\mathrm{~d}, J_{\mathrm{PC}}=34.7 \mathrm{~Hz}, \mathrm{C}\right.$-aryl); $159.4\left(\mathrm{~d}, J_{\mathrm{PC}}=16.6 \mathrm{~Hz}, \mathrm{C}\right.$-aryl). ${ }^{31} \mathrm{P}$ NMR $\left(121.4 \mathrm{MHz}, \mathrm{CDCl}_{3}\right): \delta-2.6$ (s). HRMS (ESI-Q-TOF) calcd for $\mathrm{C}_{20} \mathrm{H}_{14} \mathrm{ClN}_{2} \mathrm{PNa}[\mathrm{M}+\mathrm{Na}]^{+}$: 371.0475 , found: 371.0484 and for $\mathrm{C}_{20} \mathrm{H}_{14} \mathrm{ClN}_{2} \mathrm{OPNa}$ $[\mathrm{M}+\mathrm{O}+\mathrm{Na}]^{+}: 387.0424$, found: 387.0439 . 


\section{Acknowledgements}

The authors are grateful for the financial support provided by CNRS (Centre National de la Recherche Scientifique), the "Ministère de l'Education Nationale et de la Recherche", the "Conseil Regional de Bourgogne" (grant Pari II-smt8), and the Agence Nationale pour la Recherche (grant 07BLAN292-01 MetChirPhos). It is a pleasure for us to acknowledge for helpful discussion and technical assistance of J. Bayardon, M.J. Eymin, M.J. Penouilh, F. \& M. Picquet and B. Rugeri from the Institut de Chimie moléculaire de l'Université de Bourgogne and Welience.

\section{References}

1. Organophosphorus Chemistry, Allen. D. W.; Loakes, D.; Tebby, J. C. (Eds); RSC Publishing: Cambridge, 2012. http://dx.doi.org/10.1039/9781849734875

2. Kurihara, N.; Miyamoto, J.; Paulson, G.D.; Zeeh, B.; Skidmore, M.W.; Hollingworth, R.M.; Kuiper, H.A. Pure Appl. Chem. 1997, 69, 2007. http://dx.doi.org/10.1351/pac199769092007

3. Williams, M. L.; Wainer, I. W.; Granvil, C. P.; Gehrcke, B.; Bernstein, M. L.; Ducharme, M. P. Chirality 1999, 11, 301. http://dx.doi.org/10.1002/(SICI)1520-636X(1999)11:4<301::AID-CHIR7>3.0.CO;2-R

4. Seto, H. in Comprehensive Natural Products Chemistry, Meth-Cohn, O.; Sir Barton, D.; Nakanishi, K. (Eds); Elsevier, 1999, Vol 1, pp 865-880. http://dx.doi.org/10.1016/B978-0-08-091283-7.00032-1

5. Hayakawa, Y.; Hirabayashi, Y.; Hyodo, M.; Yamashita, S.; Matsunami, T.; Cui, D.-M.; Kawai, R.; Kodama, H. Eur. J. Org. Chem. 2006, 3834. http://dx.doi.org/10.1002/ejoc.200600155

6. Teasdale, T.; Brüggemann, O. Polymers 2013, 5, 161. http://dx.doi.org/10.3390/polym5010161

7. Levchik, S. Phosphorus-based FRs in Non-Halogenated Flame Retardant Handbook, Morgan, A.B.; Wilkie, C.A. (Eds); Scrivener Publishing LLC/Wiley, 2014, Vol 2, pp 17-74. http://dx.doi.org/10.1002/9781118939239.ch2

8. Flett, D.S. J. Organomet. Chem. 2005, 690, 2426. http://dx.doi.org/10.1016/j.jorganchem.2004.11.037

9. Phosphorus (III) Ligands in Homogeneous Catalysis: Design and Synthesis, Kamer, P.C.J.; van Leeuwen, P.W.N.M. (Eds); Wiley, 2012. http://dx.doi.org/10.1002/9781118299715

10. Rémond, E.; Jugé, S. Chemistry Today 2014, 32, 49.

11. Glueck, D.S. Top Organomet. Chem. 2010, 31, 65. 
http://dx.doi.org/10.1007/978-3-642-12073-2_4

12. Delacroix, O.; Gaumont, A.C. Curr. Org. Chem. 2005, 9, 1851. http://dx.doi.org/10.2174/138527205774913079

13. Zhao, D.; Wang, R. Chem. Soc. Rev. 2012, 41, 2095. http://dx.doi.org/10.1039/C1CS15247E

14. Enders, D.; Saint-Dizier, A.; Lannou, M.-I.; Lenzen, A. Eur. J. Org. Chem. 2006, 29. http://dx.doi.org/10.1002/ejoc.200500593

15. Join, B.; Delacroix, O.; Gaumont, A.C. Synlett 2005, 12, 1881. http://dx.doi.org/10.1055/s-2005-871576

16. Chen, Y.-R.; Duan, W.-L. Org. Lett. 2011, 13, 5824. http://dx.doi.org/10.1021/ol2024339

17. Zhao, D.; Mao, L.; Wang, L.; Yang, D.; Wang, R. Chem. Commun. 2012, 48, 889. http://dx.doi.org/10.1039/C1CC16079F

18. Chew, R.J.; Teo, K.Y.; Huang, Y.; Li, B.-B.; Li, Y.; Pullarkat, S.A.; Leung, P.-H. Chem. Commun. 2014, 50, 8768. http://dx.doi.org/10.1039/C4CC01610F

19. Carlone, A.; Bartoli, G.; Bosco, M.; Sambri, L.; Melchiorre, P. Angew. Chem. Int. Ed. 2007, $46,4504$. http://dx.doi.org/10.1002/anie.200700754

20. Terada, M; Ikehara, T.; Ube, H. J. Am. Chem. Soc. 2007, 129, 14112. http://dx.doi.org/10.1021/ja0746619

21. Zhu, Y.; Malerich, P.; Rawal, V.H. Angew. Chem. Int. Ed. 2010, 49, 153. http://dx.doi.org/10.1002/anie.200904779

22. Fritz, G.; Scheer, P. Chem. Rev. 2000, 100, 3341. http://dx.doi.org/10.1021/cr940303+

23. Hayashi, M. The Chem. Rec. 2009, 9, 236. http://dx.doi.org/10.1002/tcr.200900011

24. Hayashi, M.; Matsuura, Y.; Watanabe, Y. Tetrahedron Lett. 2004, 45, 9167. http://dx.doi.org/10.1016/j.tetlet.2004.10.098

25. Reis, A.; Dehe, D.; Farsadpour, S.; Munstein, I.; Sun, Y.; Thiel, W.R. New. J. Chem. 2011, $35,2488$. http://dx.doi.org/10.1039/C1NJ20448C

26. Reisser, M.; Maier, A.; Maas, G. Synlett 2002, 9, 1459. http://dx.doi.org/10.1055/s-2002-33533

27. Eisenberg, P.; Kieltsch, I.; Armanino, N.; Togni, A. Chem. Commun. 2008, 1575. http://dx.doi.org/10.1039/b801424h

28. Nishimura, Y.; Kawamura, Y.; Watanabe, Y.; Hayashi, M. J. Org. Chem. 2010, 75, 3875. http://dx.doi.org/10.1021/jo1004235

29. Lamas, M.-C.; Studer, A. Org. Lett. 2011, 13, 2236. http://dx.doi.org/10.1021/ol200483p 
30. Hayashi, M.; Matsuura, Y.; Watanabe, Y. J. Org. Chem. 2006, 71, 9248. http://dx.doi.org/10.1021/jo061739f

31. Trepohl, V.T.; Mori, S.; Itami, K.; Oestreich, M. Org. Lett. 2009, 11, 1091. http://dx.doi.org/10.1021/o18028466

32. Trepohl, V.T.; Fröhlich, R.; Oestreich, M. Tetrahedron 2009, 65, 6510. http://dx.doi.org/10.1016/j.tet.2009.04.038

33. Li, Y.-M.; Yang, S.-D. Synlett 2013, 24, 1739. http://dx.doi.org/10.1055/s-0033-1339341

34. Holz, J.; Zayas, O.; Jiao, H.; Baumann, W.; Spannenberg, A.; Monsees, A.; Riermeier, T.H.; Almena, J.; Kadyrov, R.; Börner, A. Chem. Eur. J. 2006, 12, 5001. http://dx.doi.org/10.1002/chem.200600033

35. Chan, V.S.; Bergman, R.G.; Toste, F.D. J. Am. Chem. Soc. 2007, 129, 15122. http://dx.doi.org/10.1021/ja076457r

36. Whittell, G.R.; Balmond, E.I.; Robertson, A.P.M.; Patra, S.K.; Haddow, M.F.; Manners, I. Eur. J. Inorg. Chem. 2010, 3967. http://dx.doi.org/10.1002/ejic.201000515

37. Chaux, F.; Frynas, S.; Laureano, H.; Salomon, C.; Morata, G.; Auclair, M-L.; Stephan, M.; Merdès, R.; Richard, P.; Ondel, M-J.; Henry, J.-C.; Bayardon, J.; Darcel, C.; Jugé, S. C.R. Chimie 2010, 13, 1213. http://dx.doi.org/10.1016/j.crci.2010.06.001

38. Bayardon, J.; Bernard, J.; Rémond, E.; Rousselin, Y.; Malacea-Kabbara, R.; Jugé, S. Org. Lett. 2015, 17, 1216 and references therein. http://dx.doi.org/10.1021/acs.orglett.5b00167

39. For Michael addition of silylphosphines with enones, see: Couret, C.; Escudié, J.; Satge, J.; Anh, N.T.; Soussan, G. J. Organomet. Chem. 1975, 91, 11.

40. Dolomanov, O.V.; Bourhis, L.J.; Gildea, R.J.; Howard, J.A.K.; Puschmann, H., OLEX2: A complete structure solution, refinement and analysis program. J. Appl. Cryst., 2009, 42, 339. http://dx.doi.org/10.1107/S0021889808042726

41 Nonius COLLECT. Nonius BV, 1997-2000, Delft, The Netherlands 1998.

42. Otwinowski, Z.; Minor, W. Methods in Enzymology 1997, 276, 307. http://dx.doi.org/10.1016/S0076-6879(97)76066-X

43. Altomare, A.; Cascarano, G.; Giacovazzo, C.; Guagliardi, A., Completion and refinement of crystal structures with SIR92, J. Appl. Cryst. 1993, 26 (3), 343. http://dx.doi.org/10.1107/S0021889892010331

44. Sheldrick, G. Acta Cryst. Sect. A 2008, 64 (1), 112. http://dx.doi.org/10.1107/S0108767307043930

45. Sheldrick, G. M. SHELX-97, Program for the Refinement of Crystal Structures, 1997, University of Göttingen, Göttingen, Germany.

46. Alonso, F.; Moglie, Y.; Radivoy, G.; Yus, M. Green. Chem. 2012, 14, 2699. http://dx.doi.org/10.1039/c2gc35898k 
47. Oswald, A.A.; Jermasen, T.G.; Westner, A.A.; Huang, I.D. US Patent 1987, 4.687.874.

48. The stereochemistry is believed to be with retention at the P-center.

49. Kumaraswamy, G.; Rao, G.V; Murthy, A.N.; Sridhar, B. Synlett 2009, 7, 1180. http://dx.doi.org/10.1055/s-0028-1088120

50. Adam, M.S.S.; Mohamad, A.D.; Jones, P.G.; Kindermann, M.K.; Heinicke, J.W. Polyhedron 2013, 50, 101.

http://dx.doi.org/10.1016/j.poly.2012.08.089 